\title{
The second French nuclear bet
}

\author{
Quentin Perrier ${ }^{\mathrm{a}, \mathrm{b}, *}$ \\ ${ }^{a}$ CIRED, 45 bis, avenue de la Belle Gabrielle, 94736 Nogent-sur-Marne Cedex, France \\ ${ }^{b}$ ENGIE, 1 place Samuel de Champlain, Faubourg de l'Arche, 92930 Paris La Défense, France
}

\begin{abstract}
Following the first oil crisis, France launched the worlds largest ever nuclear energy program, commissioning 58 new reactors. These reactors are now reaching 40 years of age, the end of their technological lifetime. This places France at an energy policy crossroads: should the reactors be retrofitted or should they be decommissioned? The cost-optimal decision depends on several factors going forward, in particular the expected costs of nuclear energy production, electricity demand levels and carbon prices, all of which are subject to significant uncertainty.

To deal with these uncertainties, we apply the Robust Decision Making framework to determine which reactors should be retrofitted. We build an investment and dispatch optimization model, calibrated for France. Then we use it to study 27 retrofit strategies for all combinations of uncertain parameters, with nearly 8,000 runs.

Our analysis indicates that robust strategies involve the early closure of 10 to 20 reactors, while extending the life of all other reactors. These strategies provide a hedge against the risks of unexpected increases in retrofit costs, low demand and low carbon prices.

Our work also highlights the vulnerabilities of the official French government scenarios, and complements them by suggesting new robust strategies. These results provide a timely contribution to the current debate on the lifetime extension of nuclear plants in France.
\end{abstract}

Keywords: Power system; Uncertainty; Nuclear costs; Robust Decision Making; Investment

JEL Classification: C6; D8; Q4

\section{Highlights}

- We estimate how many nuclear reactors should be retrofitted in France

- We identify key uncertainties and apply the framework of robust decision making

- Robust strategies imply closing 10 to 20 reactors and retrofit the others

- These results suggest a new strategy compared to the official French scenarios

\footnotetext{
${ }^{*}$ Corresponding author. E-mail address: perrier@centre-cired.fr

The opinions expressed in this paper are those of the author alone and might not represent the views of ENGIE.
} 


\section{Introduction}

The first oil shock provoked the engagement of France in an unprecedented nuclear program, which would make it up to now the country with the highest share of nuclear in its power mix. The first Contract Program was launched in 1969. A batch of six reactors of 900 MWe was ordered 5 (the CP0 batch) and their construction started in 1971. But the 1973 oil embargo triggered a more ambitious nuclear program. In early 1974, 18 identical reactors of 900 MWe were ordered (CP1 batch). They were followed in late 1975 by 18 new reactors: 10 reactors of 900 MWe (CP2 batch) and 8 of 1300 MWe (CP4 batch). 12 additional reactors of 1300 MWe were ordered in 1980 (P'4 batch). Finally, in 1984, 4 reactors of 1450 to 1500 MWe (N4 batch) concluded this nuclear policy (Boccard, 2014). These massive orders have shaped power production in France ever since. In 2015, these nuclear plants produced $78 \%$ of the country's electricity. Along with a $12 \%$ of hydropower production, it leaves only around $10 \%$ for all other sources of power (RTE) 2014).

But these power plants are now reaching the end of their lifetime. The reactors were initially designed to last for 32 years at nominal power and 40 years at $80 \%$ of their nominal power (Charpin et al. 2000, p. 31). In practice, the 40-year threshold is used by the national authority on nuclear safety, the ASN, through a system of ten-year licences. The fourth decennial inspections, which decide whether the technical lifetime can be extended beyond 40 years, are coming soon: in 2019 at Tricastin, and 2020 for Fessenheim and Bugey (Autorité de Sûreté du Nucléaire, 2014) $1{ }^{1}$

20 The lifetime of these nuclear plants can be extended if retrofit works are undertaken. A report by the French 'Cour des Comptes ${ }^{2}$ (Cour des Comptes, 2016) revealed that EDF estimates at 100 billion euros the cost of retrofitting the plants up to 2030. Should all plants be retrofitted? And more generally, what is the future of nuclear energy in France? These questions are now at the heart of public debate. The diversity of opinions on this matter was highlighted by the French

25 National Debate on Energy Transition in 2012. The views on the future of nuclear generation in France ranged from a fully maintained nuclear share, to partial retrofit strategies, to a full and early phase-out (Arditi et al., 2013).

France is now at a crossroads. The economics of this decision crucially depend on the cost of nuclear, both new and retrofitted; but these are subject to significant uncertainty. A combination

30 of recent setbacks in the nuclear industry has raised some doubt about the future cost and safety of this technology, for both the construction new nuclear reactors and the retrofitting of existing plants.

Other uncertainties must also be accounted for, in particular those concerning the level of demand and $\mathrm{CO}_{2}$ price. The level of demand can be affected by economic growth or downturn, 35 by energy efficiency and the development of new uses like electric cars. $\mathrm{CO}_{2}$ price can impact the relative competitiveness of gas and coal, but also renewable energies backed up by gas. This $\mathrm{CO}_{2}$ price depends on political will, which is difficult to anticipate.

Given these uncertainties, what are the policy options available, and what are their risks and benefits? As suggested by the current debates, there is clearly no silver bullet, no single optimum

\footnotetext{
${ }^{1}$ In practice, due to a drift in decennial inspections, the fourth decennial inspections are sometimes planned after 40 years. This drift varies among reactors and goes up to 4 years for Bugey 3. This explains why Fessenheim, although being the oldest plant, comes after Tricastin for the fourth decennial inspection.

2 The Court of Auditors (in French Cour des Comptes) is a quasi-judicial body of the French government charged with conducting financial and legislative audits of most public institutions
} 
40 strategy for all plausible values. If retrofit proves to be cheap while $\mathrm{CO}_{2}$ price and renewable costs are relatively high, then a continuation of the nuclear policy might be the cost-minimizing strategy. If nuclear is more expensive than expected and $\mathrm{CO}_{2}$ price is low, then gas turbines might be an interesting source of energy. Finally, if the cost of renewable energies keeps dropping while nuclear proves to be expensive then, with a rising $\mathrm{CO}_{2}$ price, renewable energies might become a 45 cost-effective alternative. Thus, we aim to find a robust strategy, i.e. a strategy that "performs relatively well -compared to alternatives- across a wide range of plausible futures" (Lempert et al., 2006).

From a policy point of view, it is important to define now a strategy for the medium-term and even long-term, because investment in retrofitting must be made months or years in advance. It can

50 even be ten years in advance, as the decennial stop related to the mandatory safety check is a good opportunity to start a retrofit. This implies that the future of the oldest nuclear plants must be decided soon. Proposing long-term robust strategies would contribute to the current public debate in France about the decommissioning of this plant, and about the amount of compensations to EDF if the plant were to be closed by a public decision. In the future, as new information becomes available regarding costs, demand, $\mathrm{CO}_{2}$ price or any other important parameter, the analysis should be re-runned in an iterative and flexible process.

Many studies have analysed a low-carbon transition in the power sector, at a national level (Henning and Palzer, 2015) or a European level (Jägemann et al., 2013, European Commission, 2012). Focusing more specifically on nuclear, Bauer et al. (2012) study the cost of early nuclear ${ }_{60}$ retirement at world level, and show that the additional cost of enforcing a nuclear phase-out is an order of magnitude lower that the total cost of a climate policy. But this result might not hold true for France as the share of nuclear is much higher than the world average.

Linares and Conchado (2013) analyse the break-even overnight cost of nuclear, using an optimization model of the Spanish power sector. They come up with a medium estimate of 2,609 $€ / \mathrm{kW}$, and conclude that the cost-competitiveness of this technology is questionable given the cost estimates of new projects.

In France, the French Energy Agency, ADEME, produced a study of renewable penetration in 2050 (ADEME, 2015). However, they do not study the path between today and 2050: the power mix was built ex nihilo (greenfield optimization) in 2050 as an optimization with 2050 projected 70 costs. Petitet et al. (2016) study wind penetration in France, but without modelling the potential of hydro optimization to smooth wind penetration, and consider only cases in which nuclear is cheaper than wind - a debatable assumption, as we will show. Cany et al. (2016) examined the impact of renewable penetration on the load factor and economics of French nuclear reactors, using load duration curve. They conclude on the importance of improving nuclear flexibility and on the necessity of incentives such as a carbon price for nuclear to be competitive with CCGT-gas backup. However, their approach without an optimization model does not fully capture the challenges raised by intermittent renewables, in particular regarding daily and seasonal variations.

The uncertainties of power system planning have recently been emphasized by Nahmmacher et al. (2016), using the Robust Decision Making (RDM) methodology - the same methodology that 80 we use in this paper. To our knowledge, this study by Nahmmacher et al. (2016) was the first - and the only one up to this article - in which the RDM methodology is applied to the power mix. However, their focus is different from ours: they study the resiliency of the power system to unexpected shocks, such as a transmission break-down or a heat wave. They do not deal with cost or demand uncertainty, nor with strategies of nuclear fleet management.

${ }_{85}$ In this paper, we combine the cost-minimizing optimization model of the French power sec- 
tor FLORE with the framework of Robust Decision Making (Lempert et al., 2006). This RDM framework combines scenario-based planning with statistical analysis, in order to identify robust strategies, vulnerabilities and trade-offs. We apply it to the results of nearly 8,000 runs made with the FLORE model. Our paper aims to provide insight for the current debate in France concerning the retrofit of existing nuclear plants. More generally, it also explores the importance of uncertainties related to nuclear energy in power system planning, and could thus contribute to the debates in other European countries, in particular Germany and Switzerland.

Our paper contributes to the literature in several points. The first and main one is to use a methodology of robust decision making to deal explicitly with the high uncertainty about nuclear

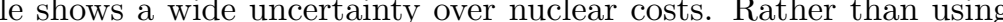
specific - and controversial - values, we propose a methodology to include this uncertainty in the decision-making process. This is an improvement compared to the pure cost-minimization, thresholds-based or sensitivity analyses. In addition, we provide new estimates on the cost of nuclear retrofit based on a novel report by the French government, and include a retrofit option in our analysis, rather than focusing on new plants. We also provide a model to study nuclear and renewables with around $12 \%$ hydro and optimized pumped storage, while the literature has focused mainly on thermal power systems, and seldom on power system with a high share of hydro (Hirth, 2016a) Linares and Conchado, 2013). Finally, we provide trajectories of nuclear for France, which is often difficult to represent properly in models covering a larger region because of its large

\section{Literature review on uncertainty ranges}

\subsection{Overview}

Various uncertainties stretch the range of plausible future costs for both retrofitted and new nuclear.

On one hand, the recent literature and events about nuclear costs presents arguments supporting the idea of increasing costs in the future:

Increased regulatory pressure on safety. Increased regulatory pressure can increase costs (Cooper 2011). In particular, the Fukushima accident has led the French nuclear regulator, the ASN, to impose new safety measures.

Unexpected cost surges. The EPR technology in Flamanville (France) has been delayed several times, and its cost tripled. For new nuclear reactors, the most famous example is the significant cost overrun of the EPR at Flamanville (France). The project was initially estimated at 3 billion euros, but this estimate has increased progressively up to 10.5 billion euros in 2015 (Garric and Bezat, 2015), and there are still concerns about the safety of its vessel (Le Hir, 2015). The EPR in Olkiluoto (Finland) is also facing several difficulties. In the UK, EDF signed a contract at $92.5 £ / \mathrm{MWh}(127 € / \mathrm{MWh}$ at 2015 exchange rate of 
Eurostat $t^{3}$ and indexed on inflation, which means the nominal price will be higher when the plant starts to produce. The exact costs of the French EPR are difficult to assess as they are not publicly displayed. This market price is thus an important clue as to the cost of this technology. All these manifestations of uncertainty raise doubts about the ability of EDF to build competitive EPR power plants.

For retrofitted nuclear, the estimation may also be optimistic, as unexpected delays often occur in such megaprojects. For example, the first retrofit, in the plant of Paluel, has led to an incident ${ }^{4}$ which may cause delays and significant cost increases (Autorité de Sûreté du Nucléaire, 2016);

Lower availability of retrofitted plants. The discovery of anomalies has led to reinforced controls. As a consequence, in October 2016, 12 reactors out of 58 were stopped to check the resistance of their steam generators (De Monicault, 2016). This lead to concerns about the security of supply and the future load factor of these plants, which is a key parameter of their value (International Energy Agency and Nuclear Energy Agency, 2015).

Risks and insurance costs. The Fukushima accident prompted a renewed interest on the question of insurance costs from the French court of audit (Cour des Comptes, 2012). This insurance should match the criticality of nuclear, i.e. the probability of an accident multiplied by severity of the consequences. Up to now, the French nuclear operator EDF has benefited from a limited liability. But both probability and damage costs are difficult to estimate. To give an idea of these uncertainties, Boccard (2014) estimates this insurance cost at $9.6 € / \mathrm{MWh}$, while Lévèque (2015) provides an expected cost below $1 € / \mathrm{MWh}$.

Decommissioning and waste management. No decommissioning has been finished yet, so the total cost is difficult to estimate. The first nuclear installation to be decommissioned in France, Brennilis, saw its cost multiplied by twenty compared to initial estimates. And in a novel report, a commission of the French Parliament reckons that the cost of dismantling might be underestimated in France (Aubert and Romagnan, 2017). As to waste management, a project called Cigeo is planned to store highly radioactive long-lived wastes. Its cost was estimated at between 15,9 and 55 billion euros in 2002 by the national radioactive waste management agency, ANDRA (Cour des Comptes, 2012, p. 142).

On the other hand, constructing several reactors of the same type can lead to learning-bydoing in the construction process. Standardization reduces lead times and thus reduces the cost of this technology. The size of reactors is also positively correlated with cost (Escobar Rangel and Lévêque, 2015), so a standardized approach with smaller reactors could thus stop the observed cost increase and even decrease the future costs of nuclear. A worldwide comparison of cost shows that cost trends vary significantly among countries, with the US experiencing strong cost escalation, while, on the other extreme, the late adopter South Korea saw a reversed trend (Lovering et al., 2016), although these results have been challenged (Gilbert et al., 2017, Koomey et al., 2017).

These elements should encourage carefulness when assessing the future costs of nuclear. They entail that a wide range of costs can be considered as plausible, in the sense that they cannot be

\footnotetext{
3 http://ec.europa.eu/eurostat/web/exchange-rates/data/main-tables

4 on March 31, 2016, a steam generator of 465 tonnes and $22 \mathrm{~m}$ high fell during its replacement operation
} 
immediately dismissed since some elements support them. In the next sections, we examine what could be the future cost of retrofitted and new nuclear, based on the current literature.

\subsection{Production cost of retrofitted nuclear plants}

\subsubsection{Historical costs}

The cost of the French nuclear reactors has been the focus of a few papers. The first official report on the historic French nuclear program was made by Charpin et al. (2000). It was later analysed by Grubler (2010), who evidenced cost escalation in time, which he coined as "negative learning-by-doing". This cost increase was then confirmed by Berthélemy and Escobar Rangel (2015). After the Fukushima accident, the French court of audits undertook a comprehensive analysis of the costs and safety of French nuclear reactors. Based on this report, Boccard (2014) revealed larger than expected operation costs for French reactors: $188 € / \mathrm{kW} /$ year, or $28.5 € / \mathrm{MWh}$ with the historical load factor. This value is a key determinant of the cost of retrofitted nuclear, since fixed capital costs are already amortized to a large extent. And this estimate is much higher than the value suggested by International Energy Agency and Nuclear Energy Agency (2015) at

$18013,3 € /$ MWh. Using the same report, Escobar Rangel and Lévêque (2015) also noticed a cost escalation, but revealed a learning curve within technologies of the same type and size.

\subsubsection{Costs of retrofitting}

These old reactors now reach the end of their planned lifetime, initially intended to be 40 year: ${ }^{5}$. These lifetimes can be extended, but only if some upgrade work is performed. A new report published by the French court of audit (Cour des Comptes, 2016) provides the costs estimated by $\mathrm{EDF}^{6}$ to retrofit its nuclear fleet and bring safety up to post-Fukushima standards. According to EDF, an investment of 100 billion euros is required by 2030: 74.73 billion euros in CAPEX and 25.16 billion euros in OPEX (Cour des Comptes, 2016). Between 2014 and 2030, 53.2 GW will reach 40 years, so the 74.73 billion euros in CAPEX yield an average additional cost of 1,404 $€ / \mathrm{kW}$.

In addition to these overnight capacity costs, the cost per unit of energy produced is influenced by capital cost, load factor and lifetime.

Financial costs are highly dependent on the rate at which capital is borrowed. In France, Quinet (2013) recommends to use a rate of $4.5 \%$ for public investments. The IEA uses a rate of $5 \%$, which we use for our best-case scenario, to account for financial costs. We use a rate of $10 \%$ for our worst-case scenario, following Boccard (2014).

For the load factor, we use a best case based on the estimates by Cour des Comptes $(2016, \mathrm{p}$. 116), which shows that the availability of French nuclear plants has been up to $83.5 \%$ since 2005 . In 2012 , the load factor was down to $73 \%$ in 2012 , but considering the recent difficulties faced by French reactors, we use a load factor of $70 \%$ as a worst case.

As to lifetime, we suppose that the investment of 100 billion euros will enable the plant to run for 20 more years. This is currently the implicit assumption of EDF (Aubert and Romagnan, 2017, p. 13), but there is significant uncertainty around this hypothesis. In France, nuclear plants are given license by periods of ten years. The French Authority of Nuclear Safety seems willing

${ }^{5}$ French reactors were initially designed for a lifetime of forty years, with a count start at their first nuclear reaction. In the US, lifetime is counted from the first layer of concrete.

${ }^{6}$ Electricité de France, the main operator of the nuclear fleet in France. 
to provide licenses for a ten-year extension; but there is no certainty that it will give a second approval, ten years later, to go beyond fifty years of operation. It may decide to close some plants, or ask for additional investment at that time. However, the magnitude of the investment indicates that EDF is confident it will be able to run for twenty more years without incurring in large additional costs. Thus, this is our best-case hypothesis. We consider a worst case in which lifetime extension will be granted for ten years only.

\subsubsection{Cost of a nuclear accident}

The Fukushima accident has prompted a renewed interest on the question of insurance costs. The insurance cost should match the criticality of nuclear, i.e. the probability of an accident multiplied by severity of the consequences. However, both the probability of an accident and damage costs are difficult to estimate.

Damage costs depend on several assumptions about indirect effects, as well as on monetizing nature and human lives (Gadrey and Lalucq, 2016). Thus, an irreducible uncertainty remains. To give an order of magnitude, the Institute for Radiological Protection and Nuclear Safety (IRSN) estimated the cost of a "controlled" release of radioactive material between 70 and 600 billion euros (Cour des Comptes, 2012), and Munich Re (2013) evaluates the damages of the Fukushima accident at 160 billion euros.

To assess the probability of a nuclear accident, two approaches coexist. The first one is the Probabilistic Risk Assessment (PRA). It consists in a technical bottom-up approach, which compounds the probabilities of failures which could lead to a core meltdown. In France, the probability 225 of an accident which would release a significant amount of radioactivity in the atmosphere is estimated at $10^{-6}$ per reactor per annum for current plants, and at $10^{-8}$ per reactor per annum for EPR reactors (Cour des Comptes, 2012, 241).

However, a wide discrepancy is observed at first glance between the expected number given by PRA estimates and the much higher number of observed nuclear accidents worldwide. This discrepancy has motivated another approach, based on the statistical analysis of past historical accidents, in particular since Fukushima. For example, Ha-Duong and Journé (2014) estimate that the historical probability of an accident was closer to $10^{-4}$ per reactor and per year - a difference of a factor 100 with PRA estimates.

However, this second approach suffers from two short-comings. The first one is the low number of occurrences. Only two events are classified in the category of major accident, i.e. a level 7 on the INES scale 7 Such a small sample hinders robust statistical analysis. Most studies thus estimate the probability of a larger pool of events by including events lower on the INES scale, but the gain in estimation accuracy comes at the cost of a change in the meaning of the results (Escobar Rangel and Lévêque, 2014). What is measured is not only the probability of major accidents occurring, but it also includes the probability of less dramatic events.

The second shortcoming comes from the use of past, historical data. This does not enable to account for the potentially increased risks due to ageing power plants, nor for the tense political situation with fears of terrorist attacks. This uncertainty can drastically change the cost of insurance. If we estimate the cost of insurance should match the criticality of nuclear, and if we assume the cost of a major nuclear accident to be 100 billion euros, as recommended by Cour des Comptes

\footnotetext{
${ }^{7}$ The INES scale, introduced by the International Nuclear Energy Agency, ranges nuclear events from 1 (anomaly) to 7 (major accident).
} 
Table 1: Estimated nuclear insurance costs for a damage cost of 100 billion euros and a load factor of 0.8

\begin{tabular}{lll}
\hline $\begin{array}{l}\text { Estimated probability of } \\
\text { an accident } \\
\text { (per reactor per annum) }\end{array}$ & $\begin{array}{l}\text { Insurance } \\
\text { (euro/MWh) }\end{array}$ & cost \\
\hline $10^{-8}$ & $1.4 \times 10^{-4}$ & \\
$10^{-6}$ & $1.4 \times 10^{-2}$ & \\
$10^{-4}$ & 1.4 \\
$10^{-3}$ & 14 & \\
\hline
\end{tabular}

(2012), then a probability of accident between $10^{-6}$ and $10^{-3}$ per reactor per annum makes the insurance cost vary from $1.4 \times 10^{-2} € / \mathrm{MWh}$ to $14 € / \mathrm{MWh}$, as shown in table 1. According to Boccard (2014), based on reports from the French 'Cour des Comptes' and the French Senate, the cost of insurance should be around $8.5 € / \mathrm{MWh}$.

The point behind these figures is that the cost of insurance cannot be dismissed once and for all. Expectations of security improvements can explain an almost negligible value for nuclear insurance, while a more conservative view can lead to significantly higher estimates.

\subsubsection{Decommissioning and waste management costs}

A novel report by the French Parliament provides several clues as to the uncertainty surrounding decommissioning costs (Aubert and Romagnan, 2017). Let us quote three. The first is related to Brennilis. This experimental reactor built in 1962, marks the first decommissioning of a nuclear power station in France. Although not achieved yet, the cost of decommissioning is currently estimated to be 482 million euros, around twenty times its initial estimate. This evolution leads to be cautious with the current cost assessments of other nuclear installations. Second, even the

260 technical feasibility of decommissioning is not granted yet. This is illustrated by the decision of EDF to postpone to 2100 the decommissioning of several experimental nuclear installations, in order to develop new decommissioning technologies. Finally, there is a large divergence between the estimates of European operators. The accounting provision is generally between 900 and 1.3 billion euros per reactor, while EDF's provision is only 350 million euros per reactor. Due to the significant difference with other European operators, we also consider a worst-case scenario in which a doubling of current costs and provisions cannot be excluded.

As to waste management, a facility called Cigeo is planned to be built to serve as a repository for highly radioactive long-lived wastes. The cost of this project was estimated at between 15,9 and 55 billion euros in 2002 by the national radioactive waste management agency, ANDRA (Cour 270 des Comptes, 2012, p. 142). Since these costs will be incurred in a distant future, valuing this nuclear liability depends on the choice of a discount rate. And this rate is subject to market fluctuations, which impacts the amount of accounting provisions and thus the cost of nuclear 8

${ }^{8}$ In France, the discount rate is currently fixed by the application decree 2007-243 of 23 February 2007 relative to ensuring the financing of nuclear accounting charges ("décret 2007-243 du 23 février 2007 relatif à la sécurisation du financement des charges nucléaires"). The nominal rate must be lower than a four-year moving average of government bonds of constant maturity at 30 years, plus a hundred base points (Cour des Comptes, 2014 p. 110). But this calculation method for the discount rate is itself debatable. For example, Taylor (2008) estimates a risk-free rate should be used. 
Given the uncertainty over costs and discounted rate, we consider another doubling of cost as our worst-case scenario.

In conclusion, decommissioning and waste management costs add another layer of uncertainty to generation costs. However, their overall impact, without being negligible, is mitigated by the discounting effects.

\subsubsection{Summing up}

Overall, there are at least eight sources of uncertainty for retrofitted reactors, as represented in figure 1 the overnight capital cost of retrofitting, the cost of borrowing, the future OPEX, the length of lifetime extension (10 or 20 years), the future availability of nuclear plants and the costs of waste, decommissioning and insurance. Using the most recent data available, detailed in table 5 and table 6 . we compute that, in the best case, the cost of retrofitted nuclear would be 44 $€ / \mathrm{MWh}$. But based on conservative assumptions detailed in table 7 in appendix, various sources of uncertainty could amount to an additional $51.5 € /$ MWh. Plausible values for this technology thus range from $44 € / \mathrm{MWh}$ to $95.5 € / \mathrm{MWh}$.

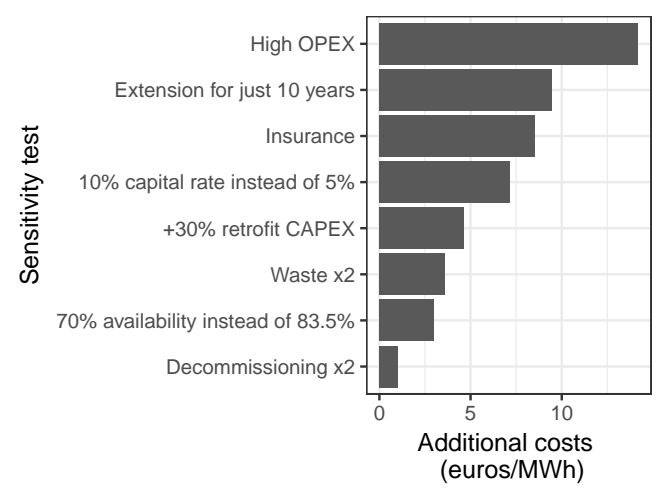

Figure 1: Plausible additional costs for retrofitted nuclear compared to a best case of $44 € / \mathrm{MWh}$. The assumptions for this sensitivity analysis are detailed in table 7 in appendix.

\subsection{Cost of new nuclear plants}

In parallel to retrofitting its existing reactors, EDF has developed a new technology: the European Pressurized Reactor (EPR). However, this model has also faced serious setbacks. The yet unfinished reactor developed in France saw its cost rising from 3 billion euros to 10.5 billion euros. In Finland, the construction work for an EPR reactor started in 2005 with a connection initially scheduled in 2009, but has so far been delayed until 2018. In the UK, the EPR has been negotiated at $92.5 £ / \mathrm{MWh}$ - approximately $127 € / \mathrm{MWh}$ at the exchange rate of 2015 given by Eurostat.

As to the future of the EPR cost, a tentative cost is estimated by Boccard (2014) between 76 and $117 € / \mathrm{MWh}$, for a total cost of 8.5 billion euros (before its upwards adjustment to 10.5 billion euros) when including back-end and insurance costs (he estimates insurance costs at around 9.6 $€ / \mathrm{MWh}$ ). The high end is in line with the contract price of the Hinkley point $\mathrm{C}$ reactor. A wide spread is found in the literature, from $76 € /$ MWh to $120 € /$ MWh. In addition, the cost of nuclear 
has been shown to depend on safety pressure. For example, Cooper (2011) showed that the Three Mile Island accident had a significant impact on cost escalation. Consequently, any new incident could increase these current estimates. This creates additional uncertainty over future nuclear costs. These significant uncertainties about future costs led some to qualify the nuclear option as a "bet" (Lévèque, 2013).

305 This wide range of nuclear cost is critical, as it encompasses the current levels of feed-in tariffs for wind in France and its LCOE 9 expected in 2050. In France, in 2014, onshore tariff was set between 55 and $82 € / \mathrm{MWh}$ for a lifetime of 20 years $(82 € / \mathrm{MWh}$ for the first ten years, and between 28 and $82 € / M W h$ afterwards, depending on wind conditions) ${ }^{10}$. Wind tariffs in neighbouring Germany were set at $59 € / \mathrm{MWh}$ in 2015: $89 € /$ MWh during 5 years and $49.5 € / \mathrm{MWh}$ afterwards Bundestag, 2014).

Finally, it is important to note that new nuclear is expected to be costlier than retrofitted plants. The general idea is that a brown field project (a retrofitted plant) requires less investment than a greenfield project. Thus, we will focus on the cases in which new nuclear is costlier than retrofitted nuclear.

\subsection{Demand, CO2 price and renewable costs}

Uncertainty in electricity demand was highlighted during the National Debate on Energy Transition (DNTE) in France, which led to four contrasted demand scenarios: SOB, EFF, DIV and DEC, in order of increasing demand. These scenarios are represented in fig. 2a. The only change we made was to halve the demand increase in the DEC scenario, because its former rate did not 320 seem not quite plausible.

These contrasted scenarios reflect the numerous uncertainties in the underlying drivers of demand. On the one hand, energy efficiency might keep improving, lowering demand (Boogen, 2017; Boogen et al. 2017). On the other hand, the increase in the number of electric vehicles or in the use of hydrogen (Cany et al., 2017) could be drivers of power demand growth. The evolution of GDP might drive demand up in case of economic expansion, or down in case of a new economic downturn. Climate change might also impact electricity demand, with higher cooling demand during summer and lower heat demand during winter Auffhammer et al., 2017; De Cian et al., 2016). Although we do not take these evolutions explicitly into account, our contrasted demand scenarios implicitly refer to such uncertainties.

${ }_{330}$ For $\mathrm{CO}_{2}$, we use the official price used in French public investments as defined in Quinet (2009). In the central scenario, this price goes up to 100 euros in 2030 to 200 euros in 2050 (see figure 2b). In the short term, these prices are significantly higher than the price of emission allowances on the EU ETS market (which has stayed below $10 € / \mathrm{tCO}_{2}$ on the EEX market in the past year) and there is no reason to think that the EU ETS price might be higher, even with the proposition 335 of back-loading some allowances in the future (Lecuyer and Quirion, 2016). We also consider a variant with medium $\mathrm{CO}_{2}$ price trajectory, in which we divide the official price by a factor of 2 ; and a low $\mathrm{CO}_{2}$ price trajectory, in which we halve the price again. These prices could represent the evolution of the EU ETS, or the price floor for $\mathrm{CO}_{2}$ currently discussed in France.

\footnotetext{
9 The Levelized Cost of Electricity, or LCOE, is an economic assessment of the average cost of one unit of energy produced by a power-generated asset. It is equal to total discounted costs divided by total discounted generation over the lifetime of the asset.

${ }^{10}$ Note that the wind developer must pay for connection to and upgrade of the distribution network to access this FIT.
} 


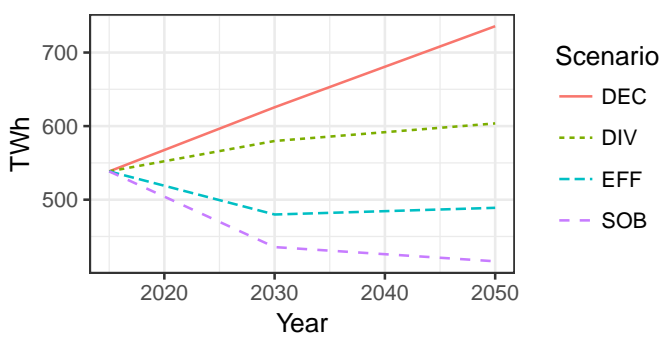

(a) Load scenarios taken from Grandjean et al. (2014).

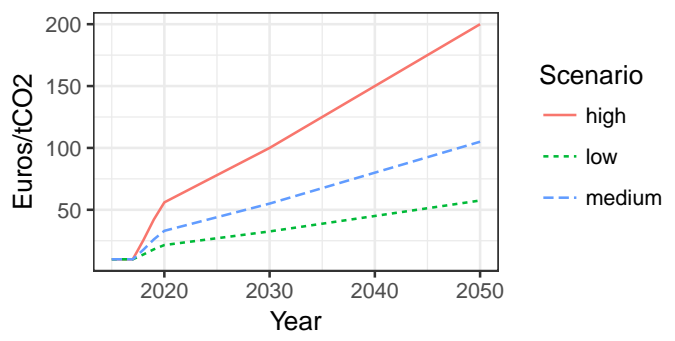

(b) $\mathrm{CO}_{2}$ price scenarios. The high end is in line with the national carbon reference price defined in Quinet (2009).

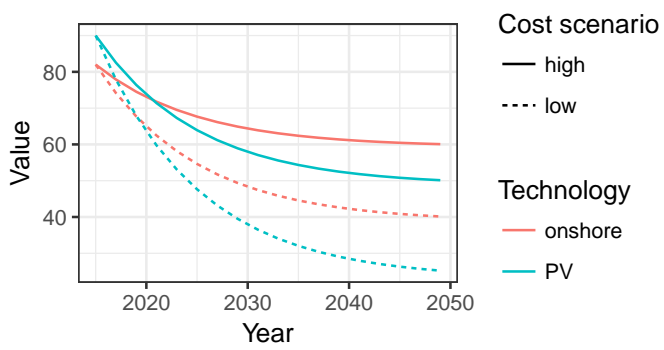

(c) Renewable energies cost scenarios. Current prices and high cost scenarios are taken from ADEME (2015). Low cost scenarios are based on recent world benchmark auction prices listed in IRENA (2017).

Figure 2: Scenarios on demand, $\mathrm{CO}_{2}$ price and renewable energies costs

All these uncertainties thus raise the question of the optimal mix. However, cost alone cannot answer that question: a model of the power mix is required. The LCOE metric does not account for the "integration costs" of variable renewable energies (VRE) (Ueckerdt et al., 2013), nor for the dispatchable nature of nuclear energy. Because PV and solar are variable, their value decreases as their penetration rate increases, although the magnitude of this decrease is specific to the power system (Hirth, 2016a) and to the renewable technology characteristics (Hirth and Müller,

2016). This phenomenon of value drop is sometimes called "self-cannibalization effect". Using an optimization model of the entire power system enables us to capture these integration costs. Indeed, since the model finds the power system that satisfies demand at the least cost to society, wind or solar would only be installed if they are competitive.

\section{Model description}

The French power model FLORE (French Linear Optimization for Renewable Expansion) is an optimization model of investment and dispatch. It is based on representative technologies, with a particular focus on nuclear and hydropower modelling to reflect the specificities of the French power mix. All technologies are endogenous for both investment and dispatch, except for hydro 
capacities for which investment is exogenous. Existing nuclear plants may be retrofitted for 20 years when they reach 40 years old, or they are decommissioned. Demand is represented by six representative weeks of 24 hours each. Hydro resources from dams and pumping stations are dispatched optimally within the representative week - but there is no annual optimization between representative weeks. In addition, all capacities are jointly optimized over the horizon, in order to provide a consistent trajectory of investments. France is represented as a single node. Hourly

360 demand, net exports and $\mathrm{CO}_{2}$ prices are exogenous. The model is calibrated for the year 2015, thus using the last generation data available from the network operator, RTE 11

All the equations of the model are available on GitHub at https://github.com/QuentinPerrier/ Flore. In addition, an interactive online version of the model can be found at http://flore. shinyapps.io/OnlineApp. This open access should ensure transparency and reproducibility, as advocated by Pfenninger et al. (2017).

\subsection{Generation technologies}

Eleven generation technologies are modelled: two variable renewable energies (onshore wind and solar PV), three fossil-based thermal technologies (coal plants, combined cycle gas turbine, open cycle gas turbine), three nuclear technologies (historical nuclear, retrofitted nuclear and new nuclear) and three hydro systems (run-of-river, conventional dams for lakes and pumped storage). Investment in each technology is a choice variable of the model, except for hydropower and historical nuclear, which are exogenous. Generation of each dispatchable technology is a choice variable. Run-of-river production is exogenous and based on historical data. Wind and solar productions are modelled as run-of-river, based on historical production profiles, with the possibility of curtailment.

375 They also incur needs for reserves, as detailed in section 3.2 .

The capacity of run-of-river and conventional dams is supposed to be constant, while the pumped hydro capacity is supposed to grow by $3.2 \mathrm{GW}$ by 2050 , with a discharge time of 20 hours, as in ADEME (2013). Hydro storage and dispatch are optimized by the model, under water reservoir constraints and pumping losses. Dams receive an amount of water to use optimally at each period. Pumped hydro can pump water (although there are losses in the process), store it into a reservoir and release the water through turbines later on.

A particularity of the model is to represent explicitly the cost of extending the lifetime of nuclear power plants. Nuclear plants normally close after 40 years, but their lifetime can be extended to 60 years, if upgrade costs are paid for. For historical nuclear, we model a phase-out of the capacity of each power plant reaching 40 years. The same year it reaches 40 years, this capacity of a historical nuclear plant is endogenously either extended for 20 years, or phased-out. Thus, each year, total investment in retrofitted plants is limited to the capacity of historical nuclear reaching forty years.

Hourly Variable Renewable Energy (VRE) generation is limited by specific generation profiles based on historical data for the year 2015. These technologies are installed whenever they are 390 competitive, although the model starts with the installed capacities of 2015 (the calibration year), which were supported by feed-in tariffs.

Dispatchable power plants produce whenever the price is above their variable costs, unless they are limited by their ramping constraints. At the end its lifetime, each capacity is decommissioned. Power generation required for heat generation was on average $2 \%$ of consumption, and never above 5\% of consumption in France in 2014 (RTE, 2014), so we do not model it.

\footnotetext{
${ }^{11}$ http://www.rte-france.com/fr/eco2mix/eco2mix-telechargement
} 


\subsection{Reserves requirements}

One concern with the penetration of variable renewable energies is the ability to meet demand at any time. As the share of VRE grows, this question of adequacy becomes a central concern for power system planning. RES forecast errors compound with other imbalances, such as demand forecast errors, and increase the need for operational flexibility. In order to provide the flexibility needed to cope with these imbalances, capacities which can rapidly add or withdraw power from the grid must be reserved: these are the reserves requirements. Modelling these reserve requirements is essential to properly evaluate the need for and the value of flexible capacities (Villavicencio, 2017).

In ENTSO-E areas, reserves are categorized into three groups: the Frequency Containment Reserves (FCR), the Frequency Restoration Reserves (FRR) and the Restoration Reserves (RR). Each group correspond to a different ramping speed. FCR must be on-line in 30 seconds. FRR restores is divided into a fast automatic component (aFRR) which must starts in 7.5 minutes, and a slow manual component (mFRR) which must be able to start in 15 minutes. Finally, RR are used to gradually replace FRR (Stiphout et al. 2016).

Since there is always a trade-off between the accuracy of technical constraints and computation time, which reserves should be considered? The expansion of renewable will put a particular emphasis on the need for FRR upwards reserve requirements. The requirements for downward reserves might be less stringent, as VRE should be able to participate in that market (Stiphout) et al., 2016; Hirth and Ziegenhagen, 2015). As to RR, ENTSO-E sets no requirement for RR (Stiphout et al., 2016) as it is not the main issue. Finally, FCR are determined by ENTSO-E for the area of Continental Europe, and this effort is then shared among the different countries. Thus, in our model, we choose to insert upwards FRR requirements and FCR. We ignore downwards FRR reserve requirements and RR. We do not model the reserves requirement for conventional power plants either.

ENTSO-E provides guidelines as to the sizing of reserve requirements for variable renewable energies. The method for sizing is well explained in Stiphout et al. (2016). It is based on a probabilistic approach of the error forecasts. The total FRR requirement is equal to the $99 \%$ quantile of the probability density function of the normalized forecast errors. It gives the required amount of capacity of FRR per unit of VRE installed capacity.

\subsection{Model resolution}

The model optimizes new capacities with a two-year time step from 2015 to 2050 . The dispatch of generation capacities is computed to meet demand for six representative weeks at an hourly step. Each typical week represents the average demand of two months of real data, through 168 hours. For example, demand of Monday for week 1 represents the average demand, on an hourly basis, of all the Mondays in January and February. This is similar to having $24 * 7=168$ time slices in the TIMES mode ${ }^{12}$ or in the LIMES-EU mode ${ }^{13}$ Demand is exogenous and assumed to be perfectly price inelastic at all times. Net export flows are given exogenously, based on historical data. The various demand scenarios we study represent the initial demand profile (domestic consumption plus exports) and change it homothetically, thus without altering the shape of peak or base demands.

The model covers France as a single region. Each typical week is associated with i) a water inflow for run-of-river and dams, ii) an availability factor for conventional generation technologies

\footnotetext{
${ }^{12} \mathrm{http}$ ///iea-etsap.org/docs/TIMESDoc-Intro.pdf

${ }^{13} \mathrm{https}$ // www.pik-potsdam.de/members/paulnah/limes-eu-documentation-2014.pdf
} 
for each representative week, to account for maintenance and iii) a production profile for renewable technologies (onshore wind and PV) based on historical production.

\subsection{Objective function}

440

The objective of the model is to minimize total system costs over the period 2015-2050. The cost of each technology is annualized, both for O\&M and capital costs. Capital annuities represent the payment of the investment loan, assuming a steady amortization plan. Using annuities allows us to avoid a border effect in 2050: the investment costs are still well represented at the end of the time horizon. This is a convenient modelling assumption, which leaves the utilization rate of

the plant endogenous in the model. However, it does not allow for early or deferred repayment of borrowed capital as could occur in reality, in case the plant has a high or low utilization rate.

We use a $0 \%$ rate of pure time preference, in order to give the same weight to the different years and generation up to 2050. This assumption is also made in the EMMA model (Hirth, 2016b) used for example by Hirth and Müller (2016). Costs include investment costs, fixed O\&M costs, variable ${ }_{450}$ costs and costs due to ramping constraints. To account for financing cost, investment costs are annualized with an $8 \%$ interest rate (the sensitivity to this parameter is included in our analysis by the fact that we vary the annualized CAPEX in our scenarios). Variable costs are determined by fuel costs, $\mathrm{CO}_{2}$ price, plant efficiency and total generation.

In section 2, we studied the range of plausible values for nuclear, highlighting eight sources of uncertainty. Looking at these specific cost items was necessary to understand the range of plausible costs for retrofitted nuclear, but we will not detail each of these items in our model, as it would lead to too many possibilities (and possibly overlapping ones). Instead, we will keep the OPEX fixed and make the annualized CAPEX vary to cover the uncertainty range. This simplified approach allows a decision maker to use its own assumptions about each cost item.

$460 \quad$ All assumptions regarding investment costs (table 9), fixed O\&M costs (table 10), $\mathrm{CO}_{2}$ price (table 11), fuel prices (table 12 and net efficiencies (table 13 can be found in appendix E

\subsection{Scope and limitations}

The objective function does not include grid costs explicitly. However, for wind farms and large PV stations, the feed-in tariffs we use include grid connection costs, as renewable producers must 465 pay for the civil engineering works required to connect the installation to distribution or transport networks (for any power exceeding $100 \mathrm{kVA}$ ).

For nuclear plants, they will be most likely built on sites with grids already built (however, the size of the new reactors is significantly higher: 1.6 GW against $0.9 \mathrm{GW}$ for many French reactors. Some upgrade words on the network might be necessary.)

Demand is exogenous. In the long-term, it means there is no price-elasticity of demand. However, we model four demand scenarios to account for demand variability.

We do not model endogenous learning curves. As we model only France, it seems like a reasonable assumption that worldwide learning curve will not be significantly impacted. However, installing more renewable energies could lead to learning at the industrial level for the installation 475 phase.

Using representative technologies entails some limits, but there is a trade-off between accuracy and runtime. Palmintier (2014) shows that using representative technologies provides errors around $2 \%$ with $\sim 1500 \mathrm{x}$ speedup compared to a fully detailed approach with Unit Commitment. Our choice implies that the investment in nuclear plants is continuous rather than constrained by steps 
of about $1 \mathrm{GW}$. To get an estimation of the number of new plants, we divide the total amount of new capacity by the average capacity of a plant.

We use only one representative technology for all historical nuclear plants, based on the relative standardization of the current fleet. This does not enable us to capture the residual heterogeneity in risks or costs between reactors (Berthélemy and Escobar Rangel, 2015). This heterogeneity

485 should be kept in mind to decide which plants should be closed first, once a given amount of capacity to decommission has been estimated by the model.

As to geographical resolution, we represent a single node and use average values. Simoes et al. (2017) shows that using a single node has a smoothing effect on renewable production, while a more accurate spatial disaggregation leads to a lower system value for both wind and solar.

490 Representing more regions would be more accurate, but also more computationally intensive. Also, the power network in France does not experience congestion problems, which justifies our single node assumption, and allows for smoothing the VRE output.

Flexibility options which could keep improving up to 2050, like demand response or storage technologies, are not represented either. Renewable technologies stay similar to today's, while there is potential for improvements in order to better integrate with the power system - e.g. with larger rotor for wind turbines (Hirth and Müller, 2016). Finally, we consider only one average profile for onshore wind and no offshore wind (but this technology is still costly and would probably not appear in an optimization model), which limits the potential benefit of spreading generation over large geographic areas. France has three different wind regimes, plus a large potential for offshore 500 wind, but our assumptions do not take fully into account that potential. This does not impact scenarios with low renewable penetration. For scenarios with high renewable penetration, including these options could help increase the value of renewables.

\section{Method: Presentation of the RDM framework}

Knight (1921) made an important distinction between risk and uncertainty. There is a risk when change can happen, for example in the value of a parameter, but the probability of any particular occurrence is measurable and known. When these probabilities are not known, there is uncertainty.

In the case of nuclear, the future costs are not known, and their probability of occurrence cannot be measured. The same holds true for demand levels, $\mathrm{CO}_{2}$ prices and renewable costs. We are dealing with a situation of uncertainty in the sense of Knight. In such situations, the traditional approach of practitioners is to employ sensitivity analysis (Saltelli et al., 2000). An optimum strategy is determined for some estimated value of each parameter, and then tested against other parameter values. If the optimum strategy is insensitive to the variations of the uncertain parameters, then the strategy can be considered a robust optimum.

However, when no strategy is insensitive to parameter changes, it is not possible to conclude to a single optimum strategy or policy. On the contrary, several optimal strategies coexist, each being contingent to the model specifications. In other words, each optimum is linked to some specific future(s).

Having a multiplicity of optimal scenarios does not enable to choose one strategy. In such a case, a decision-maker might be interested in a strategy that performs well over a large panel of futures, i.e. a robust strategy, even if it means departing from an optimal non-robust strategy. In the case of uncertainty, it means to determine a robust scenario without assuming a distribution of 
probabilities. This concept of robustness for power systems planning in face of uncertainty is not new (Burke et al., 1988, Linares, 2002), but it has received a renewed interest with the increase of computational power, and with the issue of climate change (Hallegatte, 2009).

In this paper, we use the Robust Decision Making framework developed by Lempert et al. (2006) to study the future of nuclear in France under uncertainty. This methodology was originally developed in the context of climate change. It has recently been applied to power systems - for the first time to our knowledge - by Nahmmacher et al. (2016).

The RDM framework uses the notion of regret introduced by Savage (1950). The regret of a strategy is defined as the difference between the performance of that strategy in a future state of the world, compared to the performance of what would be the best strategy in that same future state of the world. To formalize it, the regret of a strategy $s \in \mathbb{S}$ in a future state of the world $f \in \mathbb{F}$, is defined as:

$$
\operatorname{regret}(s, f)=\max _{s^{\prime}}\left\{\operatorname{Performance}\left(s^{\prime}, f\right)\right\}-\operatorname{Performance}(s, f)
$$

where $s^{\prime}$ goes through all possible strategies $\mathbb{S}$ and $\mathbb{F}$ is the set of all plausible future states. Performance is measured through an indicator, cost-minimization in our case. The regret is then equivalent to the cost of error of a strategy, i.e. the additional cost of the ex ante chosen strategy compared to the cost of what would have been the best strategy. The regret indicator allows to measure and order various strategies in each state of future. In addition, regret has the property of preserving the ranking which would result from any probability distribution. We can thus use this indicator without inferring any probability of distribution ex ante, and apply different probability distribution ex post.

The RDM method proceeds in several steps:

Determine the set of all plausible future states $\mathbb{F}$. This step aims at defining all plausible model inputs or parameter specifications. By plausible, we mean any value that cannot be excluded ex ante. The idea is to have a large set, because a set too narrow could lead to define strategies which would end up being vulnerable to some future states that were initially dismissed.

Define a set of strategies $\mathbb{S}$. These strategies will we analyzed to find a robust strategy among them. In our case, it means to define different policies as to the retrofit of nuclear plants. For example, a strategy could be to refurbish the 58 reactors; another policy, to refurbish every other reactor, and so on.

Identify an initial candidate strategy $s_{c} \in \mathbb{S}$. In RDM, a commonly used indicator is the upperquartile regret (Lempert et al., 2006, Nahmmacher et al., 2016). To get this upper-quartile regret, we compute the regret for each strategy and each future state of the world, i.e. for each tuple $(s, f) \in \mathbb{S} \times \mathbb{F}$. Then, we compute the upper-quartile regret of each strategy. In other words, for each strategy, $75 \%$ of plausible future states will be below the upper-quartile regret of that strategy. The candidate strategy is the one with the lowest upper-quartile regret.

Identify the vulnerabilities of this strategy. Here, we identify where the candidate strategy $s_{c}$ does not perform well. To do so, we find the subset of future states of the world $\mathbb{F}_{\text {vuln }}\left(s_{c}\right) \in$ $\mathbb{F}$ for which the regret is high, i.e. above some threshold value (Lempert et al. 2006). The 
value of this threshold is often defined as the upper-quartile of the regrets when considering all future states of the world (Lempert et al., 2006, Nahmmacher et al., 2016). With this definition, the vulnerabilities correspond to the future states whose regret is within the top $25 \%$. However, since this threshold value is somewhat arbitrary, it must be ensured that final results are not sensitive to the choice of this threshold. Thus, we will also consider the upper-quintile threshold to check that our results are robust. Using statistical methods (the PRIM algorithm), we then try to find what are the conditions associated with these high regret states (for example, a low demand and a high $\mathrm{CO}_{2}$ price). Finding these conditions allows to highlight the vulnerabilities of a strategy. By elimination, we can also deduct the subset $\mathbb{F}_{\text {rob }}\left(s_{c}\right)=\mathbb{F}-\mathbb{F}_{\text {vuln }}\left(s_{c}\right)$ in which the candidate strategy is robust.

Characterize trade-offs among strategies. For the subset $\mathbb{F}_{\text {vuln }}\left(s_{c}\right)$ and $\mathbb{F}_{\text {rob }}\left(s_{c}\right)$, we rank all the strategies in terms of regret, and identify the best strategy in terms of regret. Some strategies will have low regret in one subset and a high regret in the other; others will have medium regrets. It is then possible to draw a low-regret frontier, highlight the few alternative strategies on that frontier and show their trade-offs.

\section{Results}

\subsection{Optimal trajectories}

First, we study the optimal trajectories of nuclear in a cost-minimization framework, to see if there is an optimum which proves stable to sensitivity analysis, for all plausible values of uncertain parameters. In this part, we let the model choose endogenously which plants should be retrofitted.

A sensitivity analysis indicates that there is no optimum which is insensitive to the choice of input parameters. Within the plausible range, the optimal share of retrofitted nuclear varies from $0 \%$ to $100 \%$ (see appendix B). The analysis shows that the optimal share increases with the level of demand, the cost of renewable energies and the $\mathrm{CO}_{2}$ price, but decreases with the cost of retrofitted nuclear.

Hence, in this case, the framework of cost-minimization and sensitivity analysis does not allow to find a single optimum. Following our discussion in section 4. this motivates the use of the Robust Decision Making framework developed by Lempert et al. (2006). We now apply it to find robust strategies, and to highlight the potential trade-offs between various robust strategies.

\subsection{Determine plausible future states}

This step derives from the literature review in section 2. For retrofitted nuclear, we consider that plausible costs range from $40 € / M W h$ to $90 € / M W h$, by step of $10 € /$ MWh. Again, the idea is to encompass a broad initial range in order not to miss any possible value, and the RDM analysis will later identify subsets of costs to draw conclusions. As to new nuclear costs, we consider two scenarios: $90 € / \mathrm{MWh}$ and $110 € / \mathrm{MWh}$. We only take two scenarios because of the very low sensitivity to this parameter shown in the previous section 5.1

For renewable energies, we consider two trajectories: one with a quick decrease of wind and PV costs, and one with a lower decrease.

For demand, we consider four trajectories drawn from the French national debate on energy transition: a low, a decreasing, an increasing and a high trajectory. They corresponding respectively to the SOB, EFF, DIV and DEC scenario, as shown in fig. 9 . 
Finally, we examine three $\mathrm{CO}_{2}$ price trajectories: a high trajectory using the official price trajectory from Quinet (2009), and a medium trajectory in which this price is divided by two, and a low trajectory in which we halve the price again.

Overall, this combination of scenarios for costs, demand and $\mathrm{CO}_{2}$ price leads to the 288 plausible futures. Multiplied by the 27 strategies, we reach the 7,776 simulations.

\subsection{Define a set of strategies}

The next step is to define strategies of nuclear retrofit. Here, we define a strategy as the choice of which reactors to retrofit. The 58 existing reactors offer $2^{58}=2.9^{*} 10^{17}$ possibilities. As we aim to test each strategy against multiple values of uncertain parameters, testing all these possibilities would be too computationally intensive. Thus, we choose to work with a sample of strategies. This sample should represent a variability in the number (or share) of retrofitted reactors, and a variability in the time-dimension as it might be more interesting to retrofit at the beginning of the period (e.g. due higher renewable costs) or at the end (e.g. due to higher $\mathrm{CO}_{2}$ price).

To account for the time-dimension, we split the reactors into three groups: the 20 oldest reactors, the 19 medium ones, and 19 the newest ones. These groups are obtained based on chronologically-ordered cumulative capacity, divided into three thirds. This split enables to explore whether an early retrofit (or phase-out) should be preferred over a late retrofit (or phase-out).

To account for the variability in the share of retrofitted reactors, we consider three possibilities for each group: i) all reactors are retrofitted; ii) half of the reactors are retrofitted, refurbishing every other reactor from oldest to newest in the group and iii) no reactor is retrofitted.

Combining the time dimension and the retrofit share, we end up with $3^{3}=27$ different strategies.

${ }_{625}$ Figure 3 illustrates how strategies were chosen. More information on these 27 strategies can be found in appendix C. Our aim is now to find which of these strategies are robust, performing well in a wide range of futures.

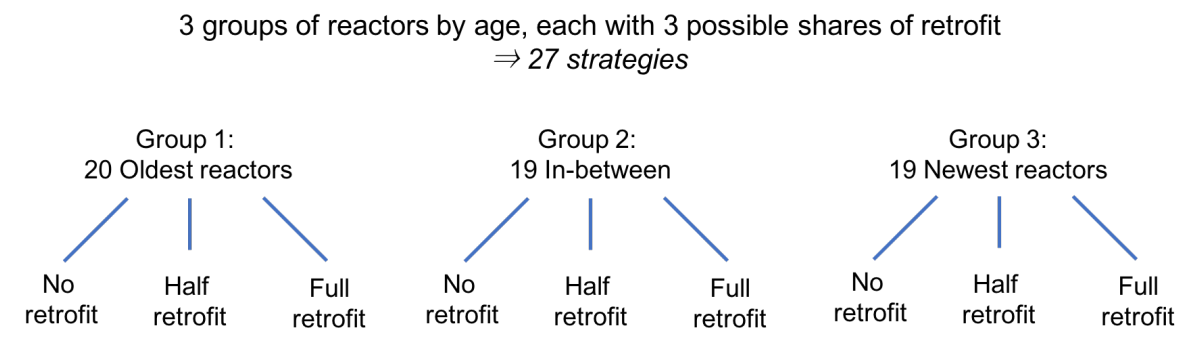

Figure 3: Defining 27 strategies

\subsection{Identify candidate strategies}

The following step is to choose a candidate strategy $s_{c}$ that performs well across plausible futures. Our indicator of performance is the upper-quartile regret. This upper-quartile regret is also the indicator used by Lempert et al. (2006) and Nahmmacher et al. (2016) to pick a candidate strategy. The idea of this indicator is to select a strategy that is not too far from the optimum in $75 \%$ of the cases, i.e. that performs well in most cases - and we will explore remaining $25 \%$ later by analyzing the vulnerabilities of our candidate strategy. 
Using the costs resulting from our model runs, we compute for each strategy $s \in \mathbb{S}$ its regret in each future state $f \in \mathbb{F}$. Then we compute the upper-quartile regret for each strategy $s$. The results are shown in fig. 4. Each dot in the figure shows the regret, in percent, for a strategy $s$ indicated on the x-axis, and a future $f$. The upper-quartile regrets are represented by the larger dots.

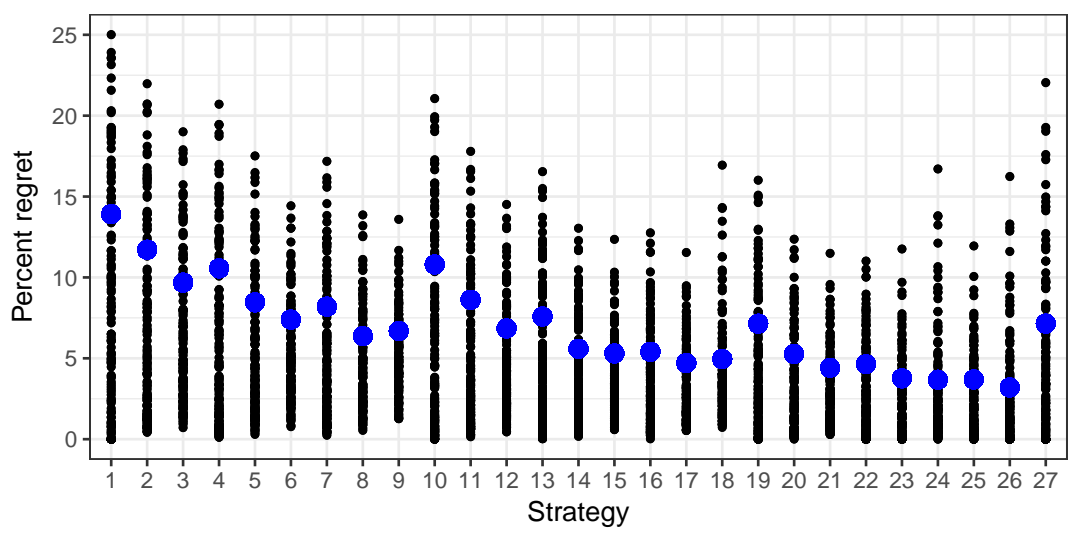

Figure 4: Each small black dot represents the regret of a strategy, indicated on the $\mathrm{x}$-axis, for one of the 288 plausible states of future considered. Details of the 288 plausible states are given in section 5.2 The larger dots, in light blue, indicate the upper-quartile regrets for each strategy. See figure 3 and appendix $\mathrm{C}$ for a definition of the 27 strategies.

The strategy with the lowest upper-quartile regret is S26 (3.2\%), and thus makes a good candidate strategy. With this strategy, ten reactors are closed, among the twenty oldest, and all the others are retrofitted.

In their paper, Lempert et al. (2006) go on with the RDM analysis considering just one candidate strategy, stating that the final results are not sensitive to the choice of the initial candidate strategy. However, we will test this assumption by performing the rest of the analysis using alternative candidate strategies. Figure 4 shows that the strategies S23, S24 and S25 are not far from S26 in terms of regret: their upper-quartile regret is $3.76 \%, 3.68 \%$ and $3.69 \%$ respectively. We thus choose to study also S23, S24 and S25 as alternative candidate strategies.

The definition of these top four candidate strategies, as well as their regret, is shown in table $650 \quad 2$

\begin{tabular}{cccccc}
\hline Strategy & 20 oldest reactors & 19 intermediate & 19 newest reactors & $\begin{array}{c}\text { Total number } \\
\text { of early closures }\end{array}$ & $\begin{array}{c}\text { Regret } \\
(\%)\end{array}$ \\
\hline S26 & half retrofit & full retrofit & full retrofit & 10 & 3.20 \\
S24 & full retrofit & half retrofit & full retrofit & 10 & 3.68 \\
S25 & no retrofit & full retrofit & full retrofit & 20 & 3.69 \\
S23 & half retrofit & half retrofit & full retrofit & 20 & 3.76 \\
\hline
\end{tabular}

Table 2: Detail of the four strategies with lowest regret 


\subsection{Identify vulnerabilities}

We keep following the RDM framework by applying the Patient Rule Induction Method (PRIM), in order to identify the vulnerabilities of our candidate strategies. This PRIM algorithm, originally been developed by Friedman and Fisher (1999), helps identify subsets of parameters in which our target indicator (regret) is above a defined threshold. Documentation on PRIM's operation can be found in Bryant and Lempert (2010). We use a public version of the PRIM algorithm implemented in $\mathrm{R}$ in the package called "sdtoolkit", available on CRAN ${ }^{14}$

Vulnerabilities correspond to the set of parameters (or futures) in which regret are too high, i.e. exceed a defined threshold. The threshold used by Lempert et al. (2006) and Nahmmacher et al. (2016) is the upper-quartile regret. With this definition, the $25 \%$ futures with highest regret will be above that threshold and considered as vulnerabilities. Although this upper-quartile is often used in the RDM approach, it is somewhat arbitrary. Thus, we will test the sensitivity of our results to this threshold by using also the upper-quintile regret.

With the upper-quartile threshold, the set of parameters associated with high regret are shown in table 3. The PRIM algorithm indicates that, for our main candidate strategy S26, the most common pattern associated with a regret above the upper-quartile regret is when retrofit costs is equal to or greater than $65 € / \mathrm{MWh}$ in conjunction with low $\mathrm{CO}_{2}$ price. These conditions provide a cluster of states of future $\mathbb{F}_{\text {vuln }}(S 26)$ to which S26 is vulnerable.

To test how these results change when selecting another candidate strategy, we apply PRIM to S23, S24 and S25. To our knowledge, this exploration of other candidate strategies has not been undertaken in other works. In our case, it appears that other clusters of vulnerabilities emerge, as indicated in table 3 . These multiple clusters indicate that there are several ways to frame the debate on nuclear in France. Each of these clusters represent a possible fault line in the debate on the best strategy, where strongly diverging options exist. The debate could focus only on nuclear 675 costs and $\mathrm{CO}_{2}$ price (with $\mathrm{S} 26$ or S24), or mostly on $\mathrm{CO}_{2}$ price (with $\mathrm{S} 23$ and $\mathrm{S} 25$ ). $\mathrm{CO}_{2}$ price is a key parameter as it defines the competitiveness of coal and gas power plants compared to nuclear and renewable energies.

Table 3: Vulnerabilities of candidate strategies

\begin{tabular}{lll}
\hline Candidate strategy & Subset of vulnerable futures & Strategies on the low-regret frontier \\
\hline $\mathrm{S} 26$ & $\begin{array}{l}\text { retrofit }>65 € / \mathrm{MWh} \\
\text { low } \mathrm{CO}_{2} \text { price }\end{array}$ & $\mathrm{S} 1, \mathrm{~S} 10, \mathrm{~S} 19, \mathrm{~S} 22, \mathrm{~S} 23$ and $\mathrm{S} 26$ \\
$\mathrm{~S} 24$ & $\begin{array}{l}\text { retrofit }>65 € / \mathrm{MWh} \\
\text { low to medium } \mathrm{CO}_{2} \text { price }\end{array}$ & $\mathrm{S} 1, \mathrm{~S} 10, \mathrm{~S} 19, \mathrm{~S} 22, \mathrm{~S} 23$ and $\mathrm{S} 26$ \\
\hline $\mathrm{S} 25$ & low to medium $\mathrm{CO}_{2}$ price & $\mathrm{S} 23$ and $\mathrm{S} 26$ \\
\hline $\mathrm{S} 23$ & low to medium $\mathrm{CO}_{2}$ price & $\mathrm{S} 23$ and S26 \\
\hline
\end{tabular}

${ }^{14}$ https://cran.r-project.org/web/packages/sdtoolkit/index.html 


\subsection{Characterize trade-offs among strategies}

Identifying vulnerabilities enables us to characterize trade-offs between different strategies. 680 Figure 5 compares the upper-quartile regret values for each of the 27 strategies over the two subsets of future states yielded by the PRIM analysis of S26: $\mathbb{F}_{\text {vuln }}(S 26)$ and $\mathbb{F}_{\text {rob }}(S 26)$. By switching from one strategy to another, it is possible to lower the regret in one cluster of states, but at the expense of increasing the regret in the other cluster of states. For example, switching from S22 to S19 reduces the regret in the vulnerable states $\mathbb{F}_{\text {vuln }}(S 26)$, but increases the regret in the other states.

Among the 27 strategies studied, some offer a better trade-off than others. In figure 5 from left to right, these are S1, S10, S19, S22, S23 and S26. These strategies are located on what can be called a Pareto-efficient or low-regret frontier. Evidencing this low-regret frontier is an important result, because it enables to select only six strategies as potential robust candidates out of the 27

690 initial strategies examined. This narrowed choice provides a simpler picture of our initial problem, showing only the strategies with interesting trade-offs.

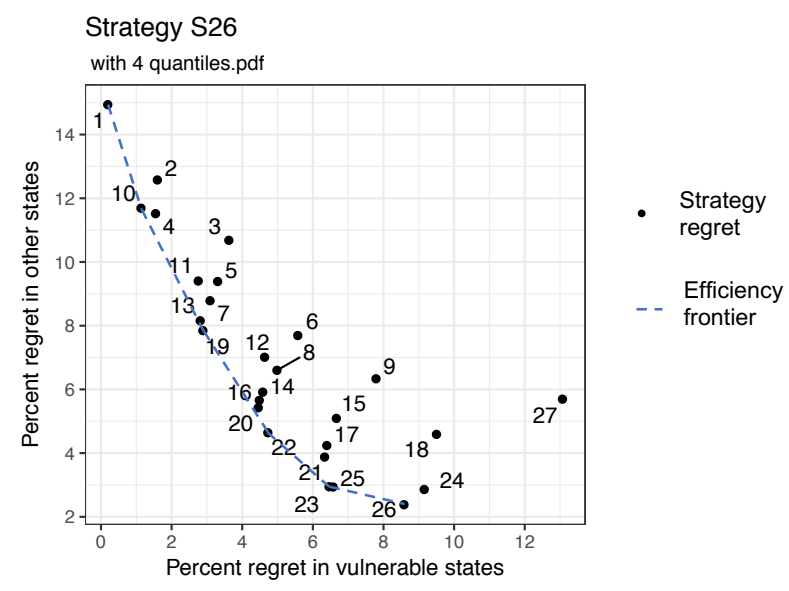

Figure 5: Regret for each strategy in $\mathbb{F}_{\text {vuln }}(S 26)$ and $\mathbb{F}_{\text {rob }}(S 26)$. The low-regret frontier is indicated by the blue dotted line.

We saw that using other candidate strategies, namely S23, S24 and S25, yields other subsets of vulnerable states. We can determine the low-regret frontier associated with these subsets. These figures are shown in fig. 7 in appendix F.1 and the strategies on these low-regret frontiers are 65 indicated in table 3

Among all of the 27 strategies, only two appear as Pareto-efficient in each of the clusters considered: S23 and S26. These two strategies represent the intersection of all the low-regret frontiers studied. For that reason, they can be considered as our robust strategies among the 27 strategies considered. S26 consists in closing 10 of the oldest reactors. S23 consists in closing 10 of the oldest reactors plus 10 intermediate reactors. In terms of cost, choosing S23 instead of $\mathrm{S} 26$ reduces regret by 2.1 percentage points in the vulnerable states of $\mathrm{S} 26, \mathbb{F}_{\text {vuln }}(S 26)$, which represents about 30 billion euros; but it increases regret by 0.56 percentage points in the robust states of $\mathrm{S} 26, \mathbb{F}_{\text {rob }}(S 26)$, which represents about 8 billion euros. 
We test the sensitivity of our results to the threshold used - the upper-quartile regret - by doing similar to the ones with the upper-quartile threshold. The main difference is that strategy S25 now also appears on the low-regret frontiers. This is not surprising, given how close S25 was to S23 in terms of regret with the upper-quartile threshold. These two strategies both imply the early decommissioning of 20 reactors, the only difference is that these closures are split between old and vulnerabilities of S23 and S25 are the same, with both upper-quartile and upper-quintile regret threshold (cf. table 14 in appendix). Thus, S25 can also be considered a robust strategy.

Closing between 10 and 20 reactors thus sketches a robust middle road, away from the two extremes of full phase-out and full retrofit. However, this result keeps the debate open, as there 715 is still a significant range of 10 reactors. Our approach has shown that these strategies (S26, S23 and S25) are on the low-regret frontier, but it does not enable us to choose between these options. The decision ultimately depends on the choice (or implicit probabilities) of the decision maker, and which vulnerabilities he would rather face. S26 is vulnerable to a conjunction of retrofitted nuclear above $65 € / \mathrm{MWh}$ and low $\mathrm{CO}_{2}$ price; $\mathrm{S} 23$ and $\mathrm{S} 25$ are vulnerable to low to medium $\mathrm{CO}_{2}$ 720 price.

In conclusion, we have evidenced that given the current estimates of cost, demand and $\mathrm{CO}_{2}$ price, the most robust strategies - among the 27 strategies considered - imply an early phase-out of at least 10 of the oldest reactors, and up to 20 reactors. The intuition behind these results comes from a balance between risks and costs. On the one hand, retrofitting all plants is a strategy share of variable renewable energies can be costly - at least in the medium term, until batteries or new renewable sources such as hydrogen become more cost-effective.

Our analysis has revealed robust strategies in-between these two extremes. Unless retrofit costs get out of hand and carbon price remains so low that it allows for a return of coal and gas power plants, retrofitting 38 to 48 reactors provides a competitive flexible source of generation. We also highlighted the vulnerabilities of these robust strategies, their alternatives, and the potential trade-offs with other strategies. Our approach keeps the debate open, as we highlight that other strategies might be considered cost-optimal for assumptions less in line with the literature, but still plausible given the current uncertainty.

\section{Comparison with French official scenarios}

\subsection{Presentation of the official scenarios}

From 2012 to 2015, France engaged in a National Debate on the Energy Transition (DNTE), which finally led to a new "Law on the Energy Transition for a Green Growth"15, This discussion on the future of the energy mix brought together various political stakeholders, as well as unions and NGOs. During the debate, the power mix and the share of nuclear were a point of particular focus, with a dedicated working group. Eleven energy transition scenarios were created by the different parties to promote their own vision of France's energy landscape, and these scenarios were ultimately grouped into four representative scenarios.

15 Loi 2015-992 du 17 août 2015 relative à la transition énergétique pour la croissance verte. 
Table 4: Description of the DNTE scenarios

\begin{tabular}{|c|c|c|c|}
\hline $\begin{array}{l}\text { Scenario } \\
\text { name }\end{array}$ & Scenario description & Demand & Nuclear Share \\
\hline $\begin{array}{l}\text { Sobriety } \\
\text { (SOB) }\end{array}$ & $\begin{array}{l}\text { A strong energy efficiency and sobriety } \\
\text { reduce power demand. } \\
\text { Nuclear and fossil fuels are phased out. }\end{array}$ & Strong decrease & Full phase-out \\
\hline $\begin{array}{l}\text { Efficiency } \\
(\mathrm{EFF})\end{array}$ & $\begin{array}{l}\text { Ambitious energy efficiency targets and } \\
\text { diversification of power sources }\end{array}$ & Slight decrease & $\begin{array}{l}\text { Decrease to } 50 \% \\
\text { in } 2030 \text { and } 25 \% \\
\text { in } 2040\end{array}$ \\
\hline $\begin{array}{l}\text { Diversification } \\
\text { (DIV) }\end{array}$ & Diversification of power sources & Slight increase & $\begin{array}{l}\text { Decrease to } 50 \% \\
\text { from } 2030 \text { on- } \\
\text { wards }\end{array}$ \\
\hline $\begin{array}{l}\text { Decarbonation } \\
\text { (DEC) }\end{array}$ & $\begin{array}{l}\text { High power demand due to increase } \\
\text { electrification and high nuclear share }\end{array}$ & Strong increase & Full retrofit \\
\hline
\end{tabular}

These four scenarios represent the different views that existed in 2012, and they have been structuring the French debate on nuclear ever since. Quantitatively, each scenario can be defined by two criteria: demand level and nuclear share. The rationale of each representative scenario is summarized in table 4 and more information can be found in fig. 9 and in Arditi et al. (2013).

\subsection{Robust vs official scenarios}

Based on this information on demand levels and nuclear shares, we can find which strategy among the 27 studied in this paper would best represent each official scenario. The DEC scenario corresponds to a retrofit of all nuclear plants. The SOB scenario is a scenario of full phase-out. Thus, their equivalent strategies are S27 and S1 respectively. But a methodology is required to determine the equivalent strategy of the EFF and DIV scenarios. To that end, we combine the information on nuclear share and demand level in each scenario to deduct the annual nuclear 755 power generation in 2020, 2030 and 2040. Generation is used as a proxy to estimate the number of retrofitted plants. For each scenario of the DNTE, we compare the nuclear production with the nuclear production in each of the 27 strategies, using the demand level corresponding to the DNTE scenario. We compute the distance using three difference norms: Euclidean, Taxicab and Infinity, in order to find the best match.

760 With this methodology, the best matches SOB and DEC are S1 (no retrofit) and S27 (full retrofit) respectively, as expected. The best match for DIV is S25 for the three norms (closing the 20 oldest reactors). The best match for EFF is S7, S11 or S13 depending on the norm, which means closing 40 reactors in each case but with variation in the timing. The correspondence between DNTE scenarios and our 27 strategies is shown in figure 10 in appendix.

765 Now that we have identified which strategy matches each scenario of the DNTE, we can build on our previous analysis and conclude that two of the DNTE scenarios offer interesting trade-offs. In particular, DIV (S25) has been identified as a robust strategy by closing 20 reactors. EFF is not a robust strategy, but it offers interesting trade-offs as it is almost on the efficiency frontier shown in figure 5. On the contrary, the two other scenarios SOB and DEC seem less robust. They 770 may provide a good option in some futures, but also risk to incur large losses in other plausible 
futures: the DEC scenario is vulnerable to a decreasing demand, and the SOB scenario bets on the high end of retrofitting cost (cf. appendix G).

In addition to evaluating the vulnerabilities of existing official scenarios, applying the RDM methodology has revealed a new option: closing about 10 of the oldest reactors. By exploring 27 initial strategies - instead of the 2 to 4 scenarios often considered in policy-making - we were able to highlight this new path, which is in-between the DIV and DEC scenarios.

The DNTE scenarios allowed an open debate with all policy options on the table, ranging from a full retrofit to an entire phase-out of all nuclear plants. Here, the search for robust strategies provides a narrower road for the years to come: closing 10 to 20 of the oldest reactors, and 780 retrofitting the rest of them. These results could contribute to the public debate in France, in particular with respect to the retrofit of the nuclear plants of Fessenheim, Bugey and Tricastin.

\section{Conclusion}

After the first oil shock, France launched the worlds largest nuclear program, ordering 36 reactors within three years, and 58 reactors in total. These reactors are now reaching the end of their technological lifetime. But extending these lifetimes requires an investment up to 100 billion euros by 2030. France is thus at a crossroads and must define a new nuclear policy. The economics of this decision crucially depend on several parameters, in particular the future costs of nuclear (including decommissioning, waste and insurance) and renewable energies, demand levels and carbon price, all of which are subject to significant uncertainty.

In face of all these uncertainties, we apply the framework of Robust Decision Making to find a robust strategy indicating which plants should be retrofitted. Based on nearly 8,000 runs and an analysis of 27 strategies, the RDM methodology revealed that the robust strategies imply the early phase-out of 10 to 20 reactors. We also assessed the vulnerabilities and alternatives of these strategies, as well as the potential trade-offs with other strategies. Our approach keeps debate open: we highlight that several options exist depending on the implicit probabilities of decision-makers. But considering the current estimates of cost, demand and carbon price, the aforementioned robust strategies seem to have the least regret.

Our work highlights the vulnerabilities of the official French government scenarios which stem from the national debate, and reveals new robust strategies. It provides a timely contribution to the debate on nuclear in France and in other countries where the decision to retrofit or decommission nuclear power plants is discussed.

Our methodology focuses on the economic costs and could be enriched by complementary analyses to include other key elements. The final decision of which power plants to close should also include safety aspects (which plants have aged better), as well as distributional and political

consideration, such as the risks of financial losses for companies, public perception (Cardin et al., 2017) and employment impacts (Garrett-Peltier, 2017). Furthermore, we only tested 27 strategies out of the $2^{58}$ possible options. Our sample was chosen to be representative of the main paths, but studying more strategies might help refine our results, and may even bring up other robust strategies.

This framework could be used iteratively in the future. Each time the decision to retrofit a nuclear plant has to be made, the same analysis could be run. The advantage would be to benefit from the latest information on costs, the level of electricity demand or the social cost of carbon. Even then, any strategy of nuclear fleet management will involve trade-offs and risks. The 
decision-maker and stakeholders should therefore pay particular attention to understanding them

and making them explicit, in order to minimize the inevitable vulnerabilities and contribute to public debate.

\section{Acknowledgements}

We would like to thank Manuel Villavicencio, Philippe Quirion, Ruben Bibas, Ibrahim Abada, Céline Guivarch, Daniel Grenouilleau, Yannick Perez, the two anonymous referees, and the participants of the 2016 FAEE workshop for their valuable comments. 
ADEME, 2013. Contribution de l'ADEME à l'élaboration de visions énergétiques 2030 / 2050. Technical Report. Ministère de l'Écologie, du Développement durable et de l'Énergie et du Ministère de l'enseignement supérieur et de la recherche.

ADEME, 2015. Un mix électrique 100\% renouvelable ? Analyses et optimisations. Technical Report. Ministère de l'Écologie, du Développement durable et de l'Énergie et du Ministère de l'enseignement supérieur et de la recherche.

Arditi, M., Durdilly, R., Lavergne, R., Trigano, É., Colombier, M., Criqui, P., 2013. Rapport du groupe de travail 2: Quelle trajectoire pour atteindre le mix énergétique en 2025 ? Quels types de scénarios possibles à horizons 2030 et 2050, dans le respect des engagements climatiques de la France? Technical Report. Rapport du groupe de travail du conseil national sur la Transition Energétique.

Aubert, J., Romagnan, B., 2017. Rapport d'information. Technical Report. Assemblée Nationale, Commission du développement durable et de l'aménagement du territoire, Mission d'information relative à la faisabilité technique et financière du démantèlement des installations nucléaires.

Auffhammer, M., Baylis, P., Hausman, C.H., 2017. Climate change is projected to have severe impacts on the frequency and intensity of peak electricity demand across the United States. Proceedings of the National Academy of Sciences 114, 1886-1891. doi:10.1073/pnas.1613193114.

Autorité de Sûreté du Nucléaire, 2014. Le calendrier prévisionnel des visites décennales des réacteurs nucléaires français. Contrôle - la revue technique de la sûreté nucléaire et de la radioprotection $198,8-9$.

Autorité de Sûreté du Nucléaire, 2016. Chute d'un générateur de vapeur dans le bâtiment du réacteur 2 de la centrale de Paluel (76) : l'ASN a diligenté n une inspection immédiate. URL: https://www.asn.fr/Informer/Actualites/ Chute-d-un-generateur-de-vapeur-1-ASN-a-diligente-une-inspection-immediate-a-Paluel-76

845 Bauer, N., Brecha, R.J., Luderer, G., 2012. Economics of nuclear power and climate change mitigation policies. Proceedings of the National Academy of Sciences 109, 16805-16810. doi:10. 1073/pnas.1201264109.

Berthélemy, M., Escobar Rangel, L., 2015. Nuclear reactors' construction costs: The role of leadn time, standardization and technological progress. Energy Policy 82, 118-130. doi:10.1016/j. enpol.2015.03.015.

Boccard, N., 2014. The cost of nuclear electricity: France after Fukushima. Energy Policy 66, 450-461. doi:10.1016/j.enpol.2013.11.037.

Boogen, N., 2017. Estimating the potential for electricity savings in households. Energy Economics 63, 288-300. doi 10.1016/J.ENEC0.2017.02.008

Boogen, N., Datta, S., Filippini, M., 2017. Demand-side management by electric utilities in Switzerland: Analyzing its impact on residential electricity demand. Energy Economics 64, 402-414. doi:10.1016/J.ENEC0.2017.04.006. 
Bryant, B.P., Lempert, R.J., 2010. Thinking inside the box: A participatory, computer-assisted approach to scenario discovery. Technological Forecasting and Social Change 77, 34-49. doi 10. $1016 / j$. techfore.2009.08.002

Bundestag, 2014. Loi pour le developpement des Energies renouven lables - EEG 2014. URL: http://www.bmwi.de/Francais/Redaktion/PDF/

10i-sur-les-energies-renouvelables-eeg-2014, property=pdf, bereich=bmwi2012, sprache $=$ fr, rwb=true.pdf.

Burke, W.J., Merrill, H.M., Schweppe, F.C., Lovell, B.E., McCoy, M.F., Monohon, S.A., 1988. Trade off methods in system planning. IEEE Transactions on Power Systems 3, 1284-1290. doi:10.1109/59.14593.

Cany, C., Mansilla, C., da Costa, P., Mathonnière, G., 2017. Adapting the French nuclear fleet to integrate variable renewable energies via the production of hydrogen: Towards massive production of low carbon hydrogen? International Journal of Hydrogen Energy 42, 13339-13356. doi:10.1016/J. I JHYDENE.2017.01.146.

Cany, C., Mansilla, C., da Costa, P., Mathonni??re, G., Duquesnoy, T., Baschwitz, A., 2016. Nuclear and intermittent renewables: Two compatible supply options? The case of the French power mix. Energy Policy 95, 135-146. doi:10.1016/j.enpol.2016.04.037.

Cardin, M.A., Zhang, S., Nuttall, W.J., 2017. Strategic real option and flexibility analysis for nuclear power plants considering uncertainty in electricity demand and public acceptance. Energy Economics 64, 226-237. doi:10.1016/J.ENEC0.2017.03.023.

Charpin, J.M., Dessus, B., Jestin-fleury, N., 2000. Étude économique prospective de la filière électrique nucléaire. Technical Report. Rapport au Premier ministre.

Cooper, M., 2011. Nuclear Safety and Nuclear Economics. Technical Report. Institute for Energy and the Environment, Vermont Law School.

Cour des Comptes, 2012. The costs of the nuclear power sector. Technical Report. Thematic public report.

Cour des Comptes, 2014. Le coût de production de l'électricité nucléaire - actualisation 2014. Technical Report. Communication à la commission d'enquête de l'assemblée nationale. Paris.

Cour des Comptes, 2016. Rapport annuel 2016 - Tome 1 - Deuxième Parte - Chapitre II : La maintenance des centrales nucléaires : une politique remise à niveau, des incertitudes à lever. Technical Report. Paris.

De Cian, E., Sue Wing, I., Wing, I.S., 2016. Global Energy Demand in a Warming Climate. Environmental and Resource Economics , 1-46doi:10.1017/CB09781107415324.004, arXiv:arXiv:1011.1669v3,

De Monicault, F., 2016. EDF va mettre à l'arrêt cinq noua veaux réacteurs. URL: http://www.lefigaro.fr/societes/2016/10/18/ 1. 20005-20161018ARTFIG00242-edf-devrait-mettre-a-l-arret-cinq-nouveaux-reacteurs. 
Escobar Rangel, L., Lévêque, F., 2014. How Fukushima Dai-ichi core meltdown changed the probability of nuclear accidents? Safety Science 64, 90-98. doi 10.1016/j.ssci.2013.11.017

Escobar Rangel, L., Lévêque, F., 2015. Revisiting the Cost Escalation Curse of Nuclear Power: New Lessons from the French Experience. Economics of Energy \& Environmental Policy 4, 103-125. doi:10.5547/2160-5890.4.2.Iran.

European Commission, 2012. Roadmap 2050. Technical Report April. URL: http://www. roadmap2050.eu/, doi:10.2833/10759, arXiv: ISBN 978-92-79-21798-2.

Friedman, J.H., Fisher, N.I., 1999. Bump Hunting in High-Dimensional Data. Statics and Computing $9,123-143$. doi: $10.1023 / \mathrm{A}: 1008894516817$.

905 Gadrey, J., Lalucq, A., 2016. Que valent les méthodes d'évaluation monétaire de la nature ? L'économie politique 69, 76-87. doi:10.3917/leco.069.0076.

Garrett-Peltier, H., 2017. Green versus brown : Comparing the employment impacts of energy efficiency, renewable energy, and fossil fuels using an input-output model. Economic Modelling 61, 439-447. doi $10.1016 / \mathrm{j}$.econmod.2016.11.012.

910 Garric, A., Bezat, J.M., 2015. EPR de Flamanville : les quatre malédictions d'un n chantier controversé. URL: http://www.lemonde.fr/economie/article/2014/11/19/

epr-de-flamanville-les-quatre-maledictions-d-un-chantier-controverse\{_\}4526032\{_\}3234. html.

Gilbert, A., Sovacool, B.K., Johnstone, P., Stirling, A., 2017. Cost overruns and financial risk in the construction of nuclear power reactors: A critical appraisal. Energy Policy 102, 644-649. doi:10.1016/J.ENPOL.2016.04.001.

Grandjean, A., Blanchet, E., Finidori, E., 2014. Etude des 4 trajectoires du DNTE. Technical Report 1. doi:10.1007/s13398-014-0173-7.2, arXiv:arXiv:1011.1669v3.

Grubler, A., 2010. The costs of the French nuclear scale-up: A case of negative learning by doing.

920 Energy Policy 38, 5174-5188. doi $10.1016 / \mathrm{j}$. enpol.2010.05.003.

Ha-Duong, M., Journé, V., 2014. Calculating nuclear accident probabilities from empirical frequencies. Environment Systems and Decisions 34, 249-258. doi 10.1007/s10669-014-9499-0.

Hallegatte, S., 2009. Strategies to adapt to an uncertain climate change. Global Environmental Change 19, 240-247. doi $10.1016 / \mathrm{j} \cdot$ gloenvcha.2008.12.003.

Henning, H.M., Palzer, A., 2015. What will the energy transformation cost? Pathways for transforming the German energy system by 2050. Technical Report. Fraunhofer Institute For Solar Energy Systems ISE. Freiburg.

Hirth, L., 2016a. The benefits of flexibility: The value of wind energy with hydropower. Applied Energy 181, 210-223. doi $10.1016 / \mathrm{j}$. apenergy.2016.07.039.

Hirth, L., 2016b. The EMMA model. Technical Report. Neon Neue Energieookonomik GmbH. URL: http://neon-energie.de/EMMA.pdf. 
Hirth, L., Müller, S., 2016. System-friendly wind power-How advanced wind turbine design can increase the economic value of electricity generated through wind power. Energy Economics 56, 51-63. doi $10.1016 / j$.eneco.2016.02.016.

Hirth, L., Ziegenhagen, I., 2015. Balancing Power and Variable Renewables: Three Links. Renewable \& Sustainable Energy Reviews 50, 1035-1051. doi:10.1016/j.rser.2015.04.180, arXiv:arXiv:1011.1669v3,

International Energy Agency, Nuclear Energy Agency, 2015. Projected costs of generating electricity. Technical Report. OECD. Paris. doi 10.1787/cost_electricity-2015-en.

IRENA, 2017. Renewable Energy Auctions : Analysing 2016. Technical Report. International Renewable Energy Agency. Abu Dhabi.

Jägemann, C., Fürsch, M., Hagspiel, S., Nagl, S., 2013. Decarbonizing Europe's power sector by 2050 - Analyzing the economic implications of alternative decarbonization pathways. Energy Economics 40, 622-636. doi $10.1016 / \mathrm{j}$.eneco.2013.08.019.

945 Knight, F., 1921. Risk, Uncertainty and Profit. Houghton m ed., Boston. doi 10.1017/ CB09781107415324.004, arXiv:arXiv:1011.1669v3

Koomey, J., Hultman, N.E., Grubler, A., 2017. A reply to Historical construction costs of global nuclear power reactors. Energy Policy 102, 640-643. doi 10.1016/J.ENPOL.2016.03.052.

Le Hir, P., 2015. L'EPR de Flamanville placé sous surveil950 lance. URL: http://www.lemonde.fr/energies/article/2015/04/16/ 1-epr-de-flamanville-place-sous-surveillance\{_\}4617161\{_\}1653054.html.

Lecuyer, O., Quirion, P., 2016. Interaction between CO2 emissions trading and renewable energy subsidies under uncertainty : feed-in tariffs as a safety net against over-allocation. FAERE Working papers 2016, 1-39.

955 Lempert, R.J., Groves, D.G., Popper, S.W., Bankes, S.C., 2006. A general, analytic method for generating robust strategies and narrative scenarios. Management Science 52, 514-528. doi $10.1287 / \mathrm{mnsc} .1050 .0472$

Lévèque, F., 2013. Nucléaire On/Off - Analyse économique d'un pari. Dunod ed.

Lévèque, F., 2015. The Economics and Uncertainties of Nuclear Power. Cambridge ed., Cambridge.

960 Linares, P., 2002. Multiple criteria decision making and risk analysis as risk management tools

口 for power systems planning. IEEE Transactions on Power Systems 17, 895-900. doi 10.1109/ TPWRS.2002.800991.

Linares, P., Conchado, A., 2013. The economics of new nuclear power plants in liberalized electricity markets. Energy Economics 40, S119-S125. doi:10.1016/j.eneco.2013.09.007.

Lovering, J.R., Yip, A., Nordhaus, T., 2016. Historical construction costs of global nuclear power reactors. Energy Policy 91, 371-382. doi:http://dx.doi.org/10.1016/j.enpol.2016.01.011.

Munich Re, 2013. Costliest natural disasters. Technical Report. Munich RE. 
Nahmmacher, P., Schmid, E., Pahle, M., Knopf, B., 2016. Strategies against shocks in power n systems an analysis for the case of Europe. Energy Economics 59, 455-465. doi $10.1016 / j$. eneco.2016.09.002.

Palmintier, B., 2014. Flexibility in generation planning: Identifying key operating constraints.

n Proceedings - 2014 Power Systems Computation Conference, PSCC 2014 doi:10.1109/PSCC. 2014.7038323 .

Petitet, M., Finon, D., Janssen, T., 2016. Carbon Price instead of Support Schemes : Wind Power. The Energy Journal 37, 109-140.

Pfenninger, S., DeCarolis, J., Hirth, L., Quoilin, S., Staffell, I., 2017. The importance of open data 口 and software: Is energy research lagging behind? Energy Policy 101, 211-215. doi $10.1016 / j$. enpol.2016.11.046.

Quinet, A., 2009. La valeur tutélaire du carbone. Technical Report. Commission présidée par Alain Quinet. Paris.

Quinet, E., 2013. L'évaluation socioéconomique des investissements publics - Tome 1. Technical Report. Rapport de la mission présidée par Emile Quinet, Commissariat général à la stratégie et à la prospective.

RTE, 2014. Eco2Mix. URL: http://www.rte-france.com/fr/eco2mix/eco2mix.

985 Saltelli, A., Chan, K., Scott, E.M., 2000. Sensitivity Analysis. New York. doi 10.1002/ 047134608X.W2510.

Savage, L.J., 1950. The Foundations of Statistics. Wiley \& Sons, New York.

Simoes, S., Zeyringer, M., Mayr, D., Huld, T., Nijs, W., Schmidt, J., 2017. Impact of different levels of geographical disaggregation of wind and PV electricity generation in large energy system

models: A case study for Austria. Renewable Energy 105, 183-198. doi 10.1016/j.renene. 2016.12.020.

Stiphout, A.V., Vos, K.D., Deconinck, G., 2016. The Impact of Operating Reserves on Investment n Planning of Renewable Power Systems. IEEE Transactions on Power Systems 8950, 1-12. doi: 10. 1109/TPWRS.2016.2565058.

Taylor, S., 2008. How should investors value nuclear liabilities? Judge Business School, University of Cambridge, Working Paper Series 4.

Ueckerdt, F., Hirth, L., Luderer, G., Edenhofer, O., 2013. System LCOE: What are the costs of variable renewables? Energy 63,61-75. doi:10.1016/j.energy.2013.10.072.

Villavicencio, M., 2017. A capacity expansion model dealing with balancing requirements, shortterm operations and long-run dynamics. CEEM Working Papers 25. 


\section{Appendix A Plausible costs of retrofitted plants}

Table 5: Historical values of French nuclear plants in the literature, in the best case

\begin{tabular}{llll}
\hline Item & Value & Unit & Source \\
\hline Historical OPEX & 13.3 & euro/MWh & Boccard, 2014 \\
Fuel & 5.7 & euro/MWh & Cour des Comptes, 2014, p. 13 \\
Waste & 3.6 & & Cour des Comptes, 2014, p. 24 \\
Decommissioning & 1 & & Cour des Comptes, 2014, p. 24 \\
Capital rate & 5 & $\%$ & \\
Lifetime extension & 20 & years & \\
availability & 83.5 & $\%$ & \\
\hline
\end{tabular}

Table 6: Retrofit costs according to EDF

\begin{tabular}{lll}
\hline Item & Value & Unit \\
\hline Additional CAPEX & 74.73 & billion euros \\
Additional OPEX & 25.16 & billion euros \\
Capacity concerned & 53.2 & GW \\
\hline
\end{tabular}

Table 7: Sensitivity tests: upper end used for nuclear costs. The variations are calculated compared to the baselines indicated in table 5

\begin{tabular}{lll}
\hline Item & Value & Unit \\
\hline CAPEX & 30 & $\%$ \\
Historical OPEX & 27.5 & euro/MWh \\
Capital rate & 10 & $\%$ \\
Lifetime extension & 10 & years \\
Availability & 70 & $\%$ \\
Waste & $\mathrm{x} 2$ & \\
Decommissioning & $\mathrm{x} 2$ & \\
Insurance & 8.5 & euro/MWh \\
\hline
\end{tabular}




\section{Appendix B Sensitivity analysis of runs with the FLORE model}

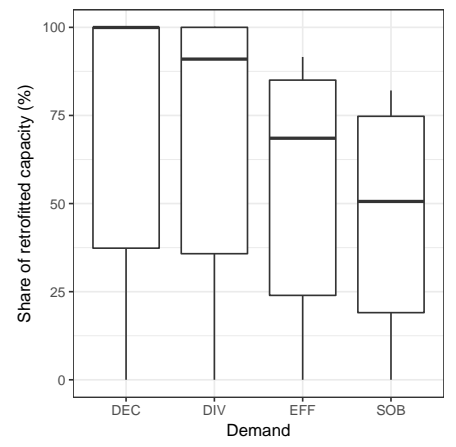

(a) Impact of demand

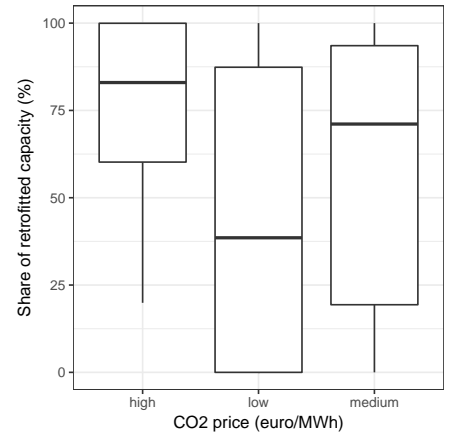

(d) Impact of $\mathrm{CO} 2$ price

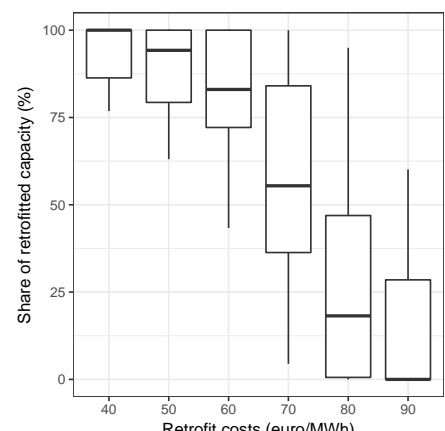

(b) Impact of retrofitting costs

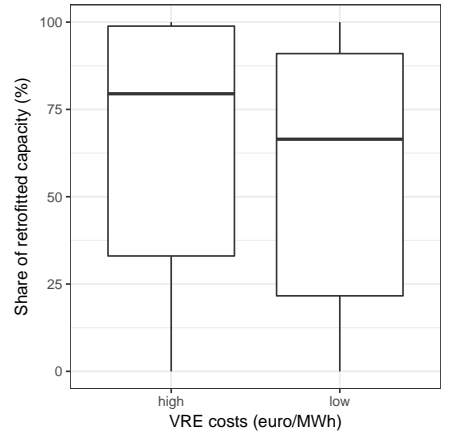

(e) Impact of VRE costs

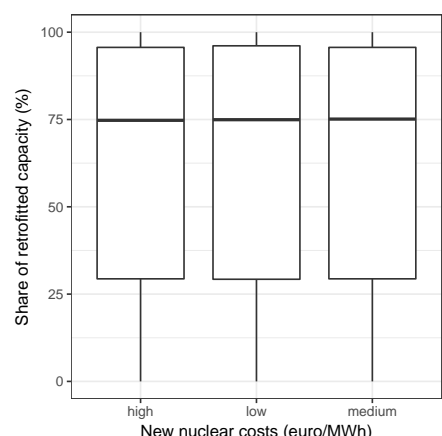

(c) Impact of new nuclear costs

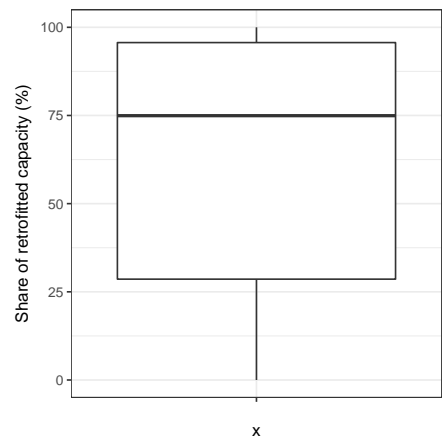

(f) Variation across all scenarios

Figure 6: Variations of the optimal share of retrofitted nuclear capacity 


\section{Appendix C Strategies examined}

Table 8: Detail of the 27 strategies

\begin{tabular}{cccc}
\hline Strategy & Oldest reactors & Intermediate reactors & Newest reactors \\
\hline S1 & no retrofit & no retrofit & no retrofit \\
S2 & half retrofit & no retrofit & no retrofit \\
S3 & full retrofit & no retrofit & no retrofit \\
S4 & no retrofit & half retrofit & no retrofit \\
S5 & half retrofit & half retrofit & no retrofit \\
S6 & full retrofit & half retrofit & no retrofit \\
S7 & no retrofit & full retrofit & no retrofit \\
S8 & half retrofit & full retrofit & no retrofit \\
S9 & full retrofit & full retrofit & no retrofit \\
S10 & no retrofit & no retrofit & half retrofit \\
S11 & half retrofit & no retrofit & half retrofit \\
S12 & full retrofit & no retrofit & half retrofit \\
S13 & no retrofit & half retrofit & half retrofit \\
S14 & half retrofit & half retrofit & half retrofit \\
S15 & full retrofit & half retrofit & half retrofit \\
S16 & no retrofit & full retrofit & half retrofit \\
S17 & half retrofit & full retrofit & half retrofit \\
S18 & full retrofit & full retrofit & half retrofit \\
S19 & no retrofit & no retrofit & full retrofit \\
S20 & half retrofit & no retrofit & full retrofit \\
S21 & full retrofit & no retrofit & full retrofit \\
S22 & no retrofit & half retrofit & full retrofit \\
S23 & half retrofit & half retrofit & full retrofit \\
S24 & full retrofit & half retrofit & full retrofit \\
S25 & no retrofit & full retrofit & full retrofit \\
S26 & half retrofit & full retrofit & full retrofit \\
S27 & full retrofit & full retrofit & full retrofit \\
\hline & & & \\
\hline
\end{tabular}




\section{Appendix D Model documentation}

\section{D.2 Total System Costs}

The model minimizes total system costs $C$ with respect to several constraints and decision variables. Total system costs are the sum of fixed costs (representing the sum of capital and fixed O\&M costs), and variable costs, over all hours $h$, weeks $w$, years $y$ and generation technologies tec.

Fixed costs are the product of the capacity installed $C A P A_{t e c, y}$ for each technology in year $y$, by its annualized capacity $\operatorname{cost} c_{t e c}^{i n v}$ plus fixed O\&M costs $c_{t e c}^{q f i x}$. Variable generation costs are the product of $G E N E_{t e c, w, y, h}$, the generation from technology tec in year $y$, week $w$ and hour $h$, by variable generation costs $c_{t e c}^{v a r}$.

$$
C=\sum_{t e c, y} C A P A_{t e c, y} \cdot\left(c_{t e c}^{i n v}+c_{t e c}^{q f i x}\right)+\sum_{t e c, y, w, h} G E N E_{t e c, y, w, h} \cdot c_{t e c}^{v a r}
$$

Throughout this model description, capital letters denote choice variable for the model, while lowercase letters denote exogenous data.

\section{D.3 Supply and demand}

Power balance between supply and demand is the central constraint of the model. Demand is the sum of national load $d$, pumping for pumped-storage hydro PUMP and exports flow flows. Supply is the sum of generation over all technologies.

$$
\forall h, y \quad \sum_{t e c} G E N E(t e c, h, y) \geq \sum_{t e c} L O A D(h, y)+P U M P(h, y)+\text { flow }(h, y)
$$


In this framework, demand is perfectly price-inelastic. Cost minimization is thus equivalent to welfare-maximization.

Generation is constrained by capacities installed and the load factor (also called availability), for all technologies except wind, PV and run-of-river hydro, which present a specific generation profile.

$$
\text { GENE(tec_noprof }, y, w, h) \leq C A P A\left(t e c \_n o p r o f, y\right) * l o a d f a c t o r\left(t e c \_n o p r o f\right)
$$

For wind and PV, a generation profile is an additional constraint:

$$
\text { GENE(tec_res, } y, w, h) \leq C A P A\left(t e c \_r e s, y\right) * r e s \_p r o f i l e s\left(h, t e c \_r e s\right)
$$

For run-of-river hydro, generation is fixed exogenously for each week:

$$
\text { GENE("river", } y, w, h)=\text { river_flow }(w)
$$

Investment is exogenous for historical nuclear and the three hydro technologies. The capacity of historical nuclear technology is decreasing based on plant-level data, with each plant capacity being phased-out when it reaches 40 years old. For hydro, we assume constant capacities for runof-river and lakes. Pumped-hydro potential is expected to grow by $3.2 \mathrm{GW}$ by 2050 , starting from 5 GW in 2014, with a discharge time of 20 hours, as in ADEME (Visions ADEME 2030).

$$
\text { CAPA(tec_ex,y) }=\text { capa_exo }\left(y, t e c \_e x\right)
$$

The capacity of all other technologies is determined endogenously by the model. Capacity is installed when optimal, and then decommissioned when it reaches its lifetime. The initial capacity, already installed in 2015, is supposed to be decommissioned linearly from 2015 onwards in a period of 40 years.

$$
\begin{array}{r}
C A P A\left(t e c \_e n d, y+1\right)=C A P A\left(t e c \_e n d, y\right)+I N V E\left(t e c \_e n d, y\right)-D E C O\left(t e c \_e n d, y\right) \\
D E C O\left(t e c \_e n d, y\right)=I N V E\left(t e c \_e n d, y-l i f e t i m e\left(t e c \_e n d\right)\right)+ \\
\left(t e c \_d a t a\left(t e c \_e n d,{ }^{\prime} \text { initcap }{ }^{\prime}\right) / 40\right) \$(\text { ord }(y)<=40)
\end{array}
$$

\section{D.4 Power System Inflexibilities}

The model includes ramp-up and ramp-down constraints for nuclear and coal power plants, to represent the fact that generation cannot vary too quickly from one hour to the next. These constraints are modelled as a maximum variation rate: The ramp up constraint is:

$$
\left.\forall t e c \_r a m p, y, w, h \quad G E N E\left(t e c \_r a m p, y, w, h+1\right) \leq G E N E\left(t e c \_r a m p, y, w, h\right) \dot{(1}+r a m p \_r a t e\right)
$$

The ramp-down constraint is:

$$
\left.\forall t e c \_r a m p, y, w, h \quad G E N E\left(t e c \_r a m p, y, w, h+1\right) \geq G E N E\left(t e c \_r a m p, y, w, h\right) \dot{(1}-r a m p \_r a t e\right)
$$

We take the value of 0.08 for coal and 0.05 for nuclear - for both ramp-up and ramp-down, as in ADEME (2015). 


\section{D.5 Reserve requirements}

We following ENTSO-E guidelines as to the sizing of reserve requirements. The method for 1040 sizing is well explained in Stiphout et al. (2016). It is based on a probabilistic approach of the error forecasts. The total FRR requirement is equal to the $99 \%$ quantile of the probability density function of the normalized forecast errors. It gives the required amount of capacity of FRR per unit of VRE installed capacity.

$$
\forall y, w, h \sum_{\text {tec_dispa }} R E S(\text { tec_dispa }, y, w, h)=\sum_{\text {tec_vre }} \epsilon(\text { tec_vre }) * C A P A\left(t e c \_v r e, y\right)
$$

Numerically, for the year 2015, we find the following values for $\epsilon$ : 0.027 for onshore, and 0.038 for PV. 


\section{Appendix E Model Calibration}

Table 9: Investment costs

\begin{tabular}{llllllllllll}
\hline euros/kW & onshore & PV & Coal & CCGT & TAC & river & lake & pump & nuc_hist & nuc_renov & nuc_new \\
\hline 2014 & 1650 & 1560 & 1500 & 800 & 400 & 3000 & 2000 & 2000 & 1320 & 1938 & 6000 \\
2020 & 1572 & 1194 & 1500 & 800 & 400 & 3000 & 2000 & 2000 & 1320 & 1938 & 6000 \\
2030 & 1443 & 869 & 1500 & 800 & 400 & 3000 & 2000 & 2000 & 1320 & 1938 & 6000 \\
2040 & 1313 & 735 & 1500 & 800 & 400 & 3000 & 2000 & 2000 & 1320 & 1938 & 6000 \\
2050 & 1184 & 600 & 1500 & 800 & 400 & 3000 & 2000 & 2000 & 1320 & 1938 & 6000 \\
\hline
\end{tabular}

Table 10: Fixed O\&M costs

\begin{tabular}{llllllllllll}
\hline euro/MWh & onshore & PV & Coal & CCGT & TAC & river & lake & pump & nuc_hist & nuc_renov & nuc_new \\
\hline 2014 & 35 & 25 & 30 & 20 & 15 & 60 & 60 & 20 & 188 & 188 & 100 \\
2020 & 35 & 25 & 30 & 20 & 15 & 60 & 60 & 20 & 188 & 188 & 100 \\
2030 & 35 & 25 & 30 & 20 & 15 & 60 & 60 & 20 & 188 & 188 & 100 \\
2040 & 35 & 25 & 30 & 20 & 15 & 60 & 60 & 20 & 188 & 188 & 100 \\
2050 & 35 & 25 & 30 & 20 & 15 & 60 & 60 & 20 & 188 & 188 & 100 \\
\hline
\end{tabular}

Table 11: $\mathrm{CO}_{2}$ price

\begin{tabular}{llll}
\hline Year & $\mathrm{CO}_{2}$ price \\
\hline \multicolumn{5}{c}{ Central } & Low & High \\
2014 & 21 & 10 & 42 \\
2020 & 28 & 14 & 56 \\
2030 & 50 & 25 & 100 \\
2040 & 75 & 38 & 150 \\
2050 & 100 & 50 & 200 \\
\hline
\end{tabular}

Table 12: Fuel prices

\begin{tabular}{llll}
\hline euro/MWh & Coal & Gas & Uranium \\
\hline 2014 & 10,3 & 25,3 & 7,30 \\
\hline
\end{tabular}

Table 13: Efficiencies

\begin{tabular}{lll}
\hline Coal & CCGT & TAC \\
\hline 0,43 & 0,61 & 0,410 \\
\hline
\end{tabular}




\section{Appendix F Analysis with PRIM}

\section{F.1 Efficiency frontier}

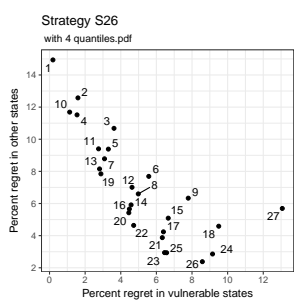

(a) With the cluster from S25

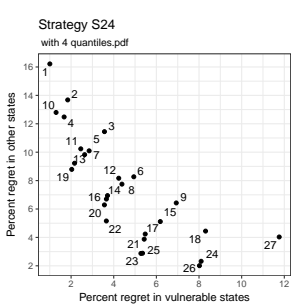

(b) With the cluster from S24 $\mathrm{\text {(c) }}$ S25

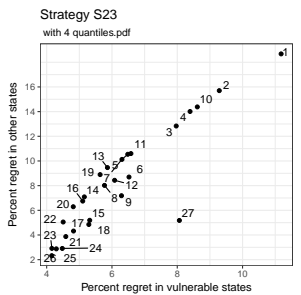

(c) With the cluster from S23 or

Figure 7: Trade-offs and efficiency frontier using the PRIM-generated clusters with upper-quartile regret

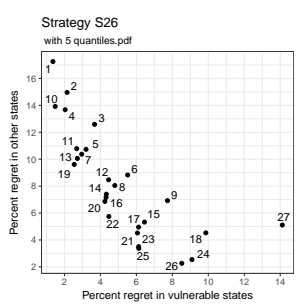

(a) With the cluster from S25

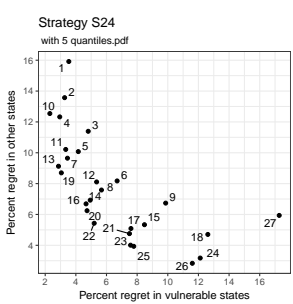

(b) With the cluster from S24 (c)

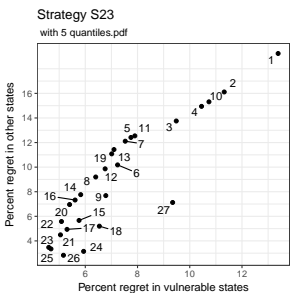

c) With the cluster from S23 or

Figure 8: Trade-offs and efficiency frontier using the PRIM-generated clusters with upper-quintile regret 


\section{F.2 Sensitivity of the threshold}

Table 14: Vulnerabilities of candidate strategies and threshold sensitivity. The italicized strategies are the strategies on the low-regret frontier

\begin{tabular}{|c|c|c|}
\hline $\begin{array}{l}\text { Candidate } \\
\text { Strategy }\end{array}$ & Upper-quartile & Upper-quintile \\
\hline $\mathrm{S} 26$ & $\begin{array}{l}\text { retrofit }>65 € / \mathrm{MWh} \\
\text { low } \mathrm{CO}_{2} \text { price } \\
S 1, S 10, S 19, S 22, S 23 \text { and } S 26\end{array}$ & $\begin{array}{l}\text { retrofit }>65 € / \mathrm{MWh} \\
\text { low to medium } \mathrm{CO}_{2} \text { price } \\
S 1, S 10, S 19, S 22, S 25 \text { and } S 26\end{array}$ \\
\hline $\mathrm{S} 24$ & $\begin{array}{l}\text { retrofit }>65 € / \mathrm{MWh} \\
\text { low to medium } \mathrm{CO}_{2} \text { price } \\
S 1, S 10, S 19, S 22, S 23 \text { and } S 26\end{array}$ & $\begin{array}{l}\text { retrofit }>65 € / \mathrm{MWh} \\
\text { low demand (SOB) } \\
\text { S10, S13, S19, S22, S23, S25 and S26 }\end{array}$ \\
\hline $\mathrm{S} 23$ & $\begin{array}{l}\text { low to medium } \mathrm{CO}_{2} \text { price } \\
\text { S23 and } S 26\end{array}$ & $\begin{array}{l}\text { low to medium } \mathrm{CO}_{2} \text { price } \\
\text { S23, S25 and S26 }\end{array}$ \\
\hline $\mathrm{S} 25$ & $\begin{array}{l}\text { low to medium } \mathrm{CO}_{2} \text { price } \\
\text { S23 and } S 26\end{array}$ & $\begin{array}{l}\text { low to medium } \mathrm{CO}_{2} \text { price } \\
\text { S23, S25 and } S 26\end{array}$ \\
\hline
\end{tabular}

\section{Appendix G Comparison with official scenarios: additional material}

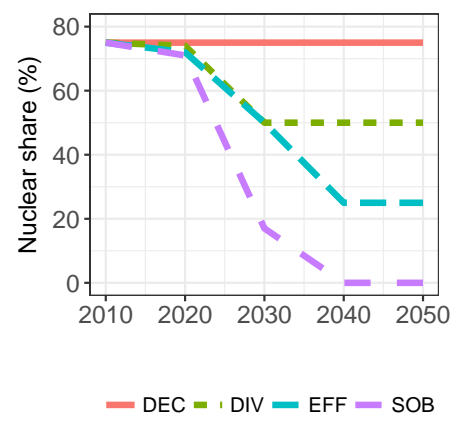

Figure 9: Nuclear share in the four official scenarios of the French national debate Source: Arditi et al. (2013)

There are several norms which can be used to evaluate the deviation between our 27 strategies and the scenarios from the DNTE. Here, we consider three of them: the Euclidean norm, the taxicab norm and the infinity norm. The results are shown in table 15 . The three norms give the same results, except for the "EFF" scenario. For this scenarios, the three norms indicates a strategy which implies to retrofit 20 reactors, but the timings vary. 


\begin{tabular}{llll}
\hline Dnte scneario & Euclidean & Taxicab & Infinity \\
\hline DEC & S27 & S27 & S27 \\
DIV & S25 & S25 & S25 \\
EFF & S13 & S7 & S11 \\
SOB & S1 & S1 & S1 \\
\hline
\end{tabular}

Table 15: Closest match, according to three norms, between the DNTE scenarios and our strategies

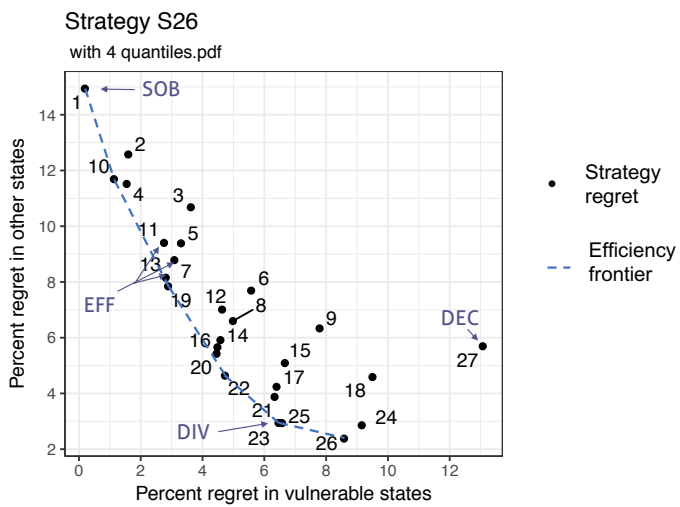

Figure 10: Correspondence between our 27 strategies and the Dnte scenarios

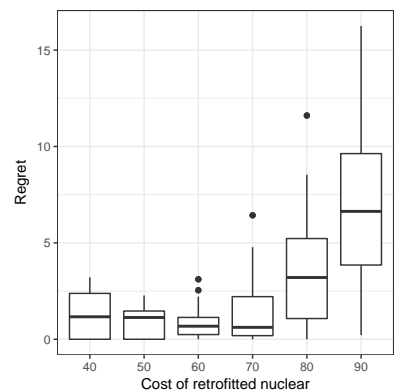

(a) Impact of retrofit cost on the SOB scenario

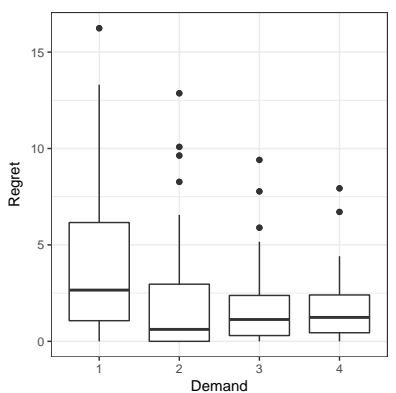

(b) Impact of demand on the DEC scenario

Figure 11: Vulnerabilities of DNTE scenarios 